\title{
A SOLUTION TO THE CIRCULAR RESTRICTED N BODY PROBLEM IN PLANETARY SYSTEMS
}

\author{
A Thesis \\ presented to \\ the Faculty of California Polytechnic State University, \\ San Luis Obispo
}

\author{
In Partial Fulfillment \\ of the Requirements for the Degree \\ Master of Science in Aerospace Engineering
}

by

Jay R. Iuliano

June 2016 
(C) 2016

Jay R. Iuliano

ALL RIGHTS RESERVED 


\section{COMMITTEE MEMBERSHIP}

TITLE:

A Solution To The Circular Restricted N

Body Problem In Planetary Systems

AUTHOR: Jay R. Iuliano

DATE SUBMITTED: June 2016

COMMITTEE CHAIR: Kira Abercromby, Ph.D.

Associate Professor of Aerospace Engineering

COMMitTeE MEMBER: Eric Mehiel, Ph.D.

Professor of Aerospace Engineering

COMMITTEE MEMBER: Ian Johnson, Ph.D.

Lecturer of Aerospace Engineering

COMmitTeE MeMBeR: Paul Choboter, Ph.D.

Associate Professor of Mathematics 


\begin{abstract}
A Solution To The Circular Restricted N Body Problem In Planetary Systems
\end{abstract}

Jay R. Iuliano

This thesis is a brief look at a new solution to a problem that has been approached in many different ways in the past - the $\mathrm{N}$ body problem. By focusing on planetary systems, satellite dynamics can be modeled in a fashion similar to the Circular Restricted Three Body Problem (CR3BP) with the Circular Restricted N Body Problem (CRNBP). It was found that this new formulation of the dynamics can then utilize the tools created from all the research into the CR3BP to reassess the possibility of different complex trajectories in systems where there are more than just two large gravitational bodies affecting the dynamics, namely periodic and semi-periodic orbits, halo orbits, and low energy transfers It was also found that not only system dynamics, but models of the Jacobi constant could also be formulated similarly to the CR3BP. Validating the authenticity of these new sets of equations, the CRNBP dynamics are applied to a satellite in the Earth-Moon system and compared to a simulation of the CR3BP under identical circumstances. This test verified the dynamics of the CRNBP, showing that the two systems created almost identical results with relatively small deviations over time and with essentially identical path trends. In the Jovian system, it was found the mass ratio required to validated the assumptions required to integrate the equations of motion was around $.1 \%$. Once the mass ratio grew past that limit, trajectories propagated with the CRNBP showed significant deviation from trajectories propagated with a higher fidelity model of Newtonian motion. The results from the derivation of the Jacobi constant are consistent with the 3 body system, but they are fairly standalone. 


\section{ACKNOWLEDGMENTS}

Thank you to my friends and colleagues, who have always encouraged me, and a very special thank you to my professors, without their incredible patience for my ridiculous ideas, I would never have made it this far. 


\section{TABLE OF CONTENTS}

LIST OF TABLES . . . . . . . . . . . . . . . . . . . . . . . . . . . . . . . . . . . vii vii
LIST OF FIGURES
CHAPTER

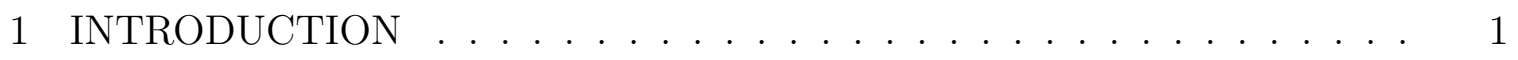

1.1 Background ........................... 1

2 THE CIRCULAR RESTRICTED N BODY PROBLEM . . . . . . . . . . 12

2.1 Derivation of the Equations of Motion . . . . . . . . . . . 12

2.1.1 Test Cases ....................... 14

2.2 The Jacobi Constant . . . . . . . . . . . . . . . . . . . . . 22

2.2.1 Test Cases . . . . . . . . . . . . . . . . . 25

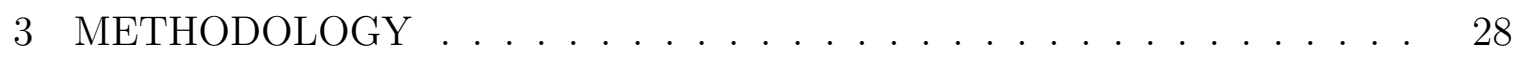

3.1 Modeling Motion . . . . . . . . . . . . . . 28

3.2 Calculating the Jacobian Energy and Characterizing Stability . . . . 29

3.3 Manifold Investigation . . . . . . . . . . . . . . . . 30

4 RESULTS . . . . . . . . . . . . . . . . . . . . 32

4.1 Stable Regions, and Quasi-stable orbits . . . . . . . . . . . . . . 34

4.2 Halo Orbits and Manifolds . . . . . . . . . . . . . . . . . 41

4.3 Low Energy Transfers and the Possibility of Highways . . . . . . . . . 54

5 FUTURE WORK . . . . . . . . . . . . . . . . . . 59

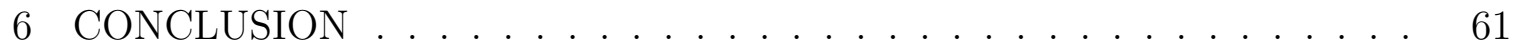

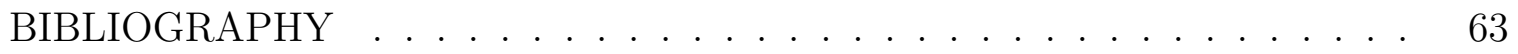




\section{LIST OF TABLES}

Table

Page

4.1 Canonical Conversions . . . . . . . . . . . . . . . . 33 


\section{LIST OF FIGURES}

Figure

Page

1.1 Basic setup for the CR3BP . . . . . . . . . . . 4

1.2 Energy curves and Lagrange points in the Earth-Moon CR3BP . . 6

1.3 A Family of Lissajous Halo Orbits . . . . . . . . . . . . . 8

1.4 Stable (left) and Unstable (right) manifolds . . . . . . . . . . . 9

2.1 Basic setup for the CRNBP . . . . . . . . . . . . . . 13

2.2 Trajectory comparisons between the CRNBP (red) and the CR3BP (blue). Both are given the same starting conditions . . . . . . . . 15

2.3 A test trajectory with quasi-stable trans-lunar orbiting properties. The plot on the left has the energy curves of the system laid over top of it . . . . . . . . . . . . . . . . 15

2.4 Comparison to High Fidelity Propagation _. . . . . . . . . . 18

2.5 Divergent Comparison to High Fidelity Propagation . . . . . . . . . 19

2.6 Long Duration Comparison to High Fidelity Propagation . . . . . . 19

2.7 Mass Ratio Tests $(\mathrm{x} 2$ and $\mathrm{x} 5)$. . . . . . . . . . . . 20

2.8 Mass Ratio Tests (x10 and x100) . . . . . . . . . . . . 21

2.9 Two and three dimensional plots of the Jacobi energy for the Jovian system .......................... 26

2.10 Jacobi Energy near Ganymede and Callisto . . . . . . . . . . 26

2.11 Jacobi Energy near Io and Europa . . . . . . . . . . . . . . . 27

4.1 Motion of the "Pseudo-Lagrange" Jupiter-Ganymede L1 point for 1 Week ......................... 36

4.2 Motion of the "Pseudo-Lagrange" Jupiter-Ganymede L1 point for an extended period . . . . . . . . . . . . . . . . . 37

4.3 Examples of quasi-periodic orbits . . . . . . . . . . . . . 38

4.4 Out-of-plane quasi-periodic orbits . . . . . . . . . . . . 38

4.5 Planar periodic orbits . . . . . . . . . . . . . . 39

4.6 Trans-Ganymede Periodic Orbit . . . . . . . . . . . . . . . 40

4.7 A highly irregular orbit with unique viewing coverage properties . . 41

4.8 Corrected quasi-halo orbiting . . . . . . . . . . . 43 


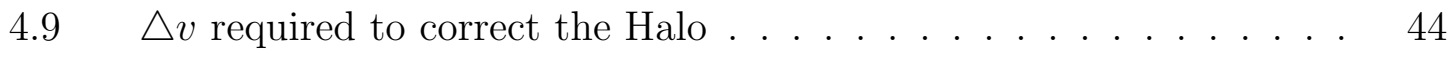

4.10 Corrected quasi-halo orbiting with wobbling . . . . . . . . 44

$4.11 \Delta v$ for the wobbling halo $\ldots \ldots \ldots \ldots \ldots$

4.12 Unstable corrected quasi-halo orbiting . . . . . . . . . . . 46

$4.13 \triangle v$ for the unstable halo . . . . . . . . . . . . . . 46

4.14 Divergent corrected quasi-halo orbiting . . . . . . . . . . 47

$4.15 \Delta v$ for the divergent halo $\ldots \ldots \ldots \ldots \ldots$

4.16 Highly stable corrected quasi-halo orbiting . . . . . . . . . 48

$4.17 \triangle v$ for the stable halo $\ldots \ldots \ldots \ldots$

4.18 Convergent corrected quasi-halo orbiting . . . . . . . . . . 49

$4.19 \triangle v$ for the convergent halo . . . . . . . . . . . . . 49

4.20 Manifolds propagated with 3 body dynamics from halos developed with 3 body dynamics . . . . . . . . . . . . . . . . 50

4.21 Manifolds propagated with $\mathrm{N}$ body dynamics from a halo developed with 3 body dynamics . . . . . . . . . . . . . . . . . 51

4.22 Manifolds propagated with $\mathrm{N}$ body dynamics from halos developed with 3 body dynamics near Callisto . . . . . . . . . . . . . . 52

4.23 Manifolds propagated with $\mathrm{N}$ body dynamics from halos developed with 3 body dynamics near Europa and Io . . . . . . . . . . . . . 53

4.24 Manifolds propagated with $\mathrm{N}$ body dynamics from a halo developed with $\mathrm{N}$ body dynamics . . . . . . . . . . . . . 53

4.25 Low Energy Transfer into the Jovian System . . . . . . . . . . 55

4.26 Low Energy Transfer into the Jovian System (zoom) . . . . . . . 56

4.27 Transfer from Sun-Jupiter L1 Halo to Jupiter-Ganymede L2 Halo

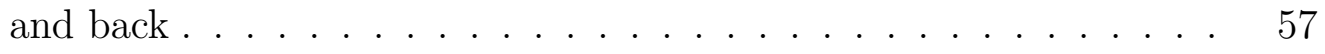

4.28 Transfer from Sun-Jupiter L1 Halo to Jupiter-Ganymede L2 Halo and back (zoom) . . . . . . . . . . . . 57 
Chapter 1

\section{INTRODUCTION}

\subsection{Background}

Modeling the motion of bodies under the influence of gravity is a non-trivial task as soon as you get past the most basic form of Kepler two body motion. Perturbations from other gravitational bodies, bodies with unevenly distributed mass, pressure from radiation and gases, and even tidal flow make the reliable simulation and tracking of objects in orbit difficult. Countless papers, methods, and tricks have been published and practiced over the last century to create more reliable and effective models, yet there still remains a large number of unsolved problems in the world of orbital mechanics. One of the most interesting and prevalent discussions is that of $\mathrm{N}$ body motion. Put more clearly, the question "What is the best way to simulate the trajectory of small bodies under the influence of several large bodies?" has not yet been given a great answer, especially not one that can be applied generically.

The first and perhaps the most brutish way to attack difficult orbital problems is simply to create extremely high fidelity models, models that include every possible perturbation. The problems with this are obvious, the largest being computation time. It is often unnecessary to model slight changes in an orbit if your results are subject to random error on the order of or larger than the perturbation itself. For example, while it might be pertinent to include the effects of relativity if you are trying to model the collision of galaxies, doing so on the scale of satellites would be a waste of time. Even if all unnecessary considerations are removed, having a simulation that is very high fidelity can increase computation time through arbitrary calculation. Take, for example, the method of averaging perturbations for low thrust transfers. 
By assuming that the rate of change of right ascension, argument of perigee, and true anomaly are represented over long periods by a given average rate of change, you have effectively cut your simulation time in half despite the slight loss in accuracy Much in the same way that one cannot always average out the changes in certain orbital elements in high accuracy models, to say that one way of modeling $\mathrm{N}$ body motion is better requires an understanding of the conditions under which the model is valid. Perhaps it's the more apparent, then, that the question "What is the best way to simulate the trajectory of small bodies under the influence of several large bodies?" requires knowledge of the specific set of conditions the trajectory needs to be modeled.

To explore the answers to this problem, and to investigate the possibility of a different solution to the $\mathrm{N}$ body problem in planetary systems specifically is the goal of this thesis. The first step in completing this task is to decide out which method is most appropriate for modeling the motion of satellites in the planetary systems with multiple large bodies in close vicinity. The $\mathrm{N}$ body problem has been attacked in numerous ways for a variety of different scenarios. Astronomers have a number of different methods for simulating the motion of $\mathrm{N}$ bodies when $\mathrm{N}$ is greater than 1000 such as in the case of galaxy formation or collision. This involves the well known Leap-Frog integration technique by which positions of bodies are updated via their previous position and their projected velocity at a time between the previous time step and the current one[4]. While the integration method is a generally successful method, its main benefit is its mathematical stability through the integration process which is unnecessary when the number of bodies is small. These methods are designed for enormous numbers of bodies and there are no planetary systems like that in our solar system. Even Jupiter, with its seemingly endless supply of moons, has only 4 moons that have any significant gravitational impact. Together, the Galilean moons make up $99.997 \%$ of the mass of all of Jupiter's orbiting bodies. 
The most classic method of simulating $\mathrm{N}$ body effects is to take classic Kepler two body motion and to treat the gravity from each additional body as a small deviation from that motion. The method of patched conics simply ignores extra bodies and assumes that the motion of a small body can be approximated by 2 body motion around the body with the largest gravitational influence. As soon as a satellite (satellite is synonymous for a small body with no notable gravitational impact throughout this paper) leaves the sphere of influence of one body, it is assumed to no longer be affected by the first body at all[2]. The problem with this method is that it doesn't properly account for scenarios in which the sphere of influence of one body is contained inside the sphere of influence of another body, such as is the case with the Earth and its moon. In this case, one of the best ways to model the motion of a satellite is with the solutions to the planar circular restricted three body problem.

The Planar Circular Restricted 3 Body Problem (CR3BP) reveals a solution to modeling the dynamics of a satellite in a gravitational systems with 2 large gravitational bodies. This solution allows for the analysis of systems like the Earth-Moon system with relative ease and without the computationally expensive task of high fidelity simulations. The solutions to this problem also open the door to cornucopia of interesting trajectories, including low energy transfers, which mitigate otherwise huge $\triangle v$ costs of traditional transfers, stable repeating orbits that cover large swathes in the sky with convenient and moldable coverage properties, and rapid propagation of large, long duration orbits that would otherwise be impractical to study, let alone to calculate on standard household computers and laptops. Particularly useful are halo orbits and manifolds which act as possible starting points for interplanetary transfers and high-scope observational missions. For these reasons, this model was the starting point for the solutions to the problem that this thesis is focused on. Because this thesis depends so heavily on the ability to recreate the solutions to this problem (in a different form), it is important that the CR3BP is well understood before diving 
into the actual derivations and results of the paper.

The CR3BP focuses on the motion of a satellite around the barycenter of a given gravitational system without assuming that the barycenter of the system is centered on the largest body as is done in Kepler two body motion. The general setup for the system is given below in Figure 1.1, where $\mu^{*}$ represents the mass ratio of the large bodies in the system. If $m_{2}$ is the smaller body, $\mu^{*}=\frac{m_{2}}{m_{1}+m_{2}}$. In this figure, the mass of the satellite is given as $m_{3}$, and distances are normalized to the distance between the two largest bodies[11].

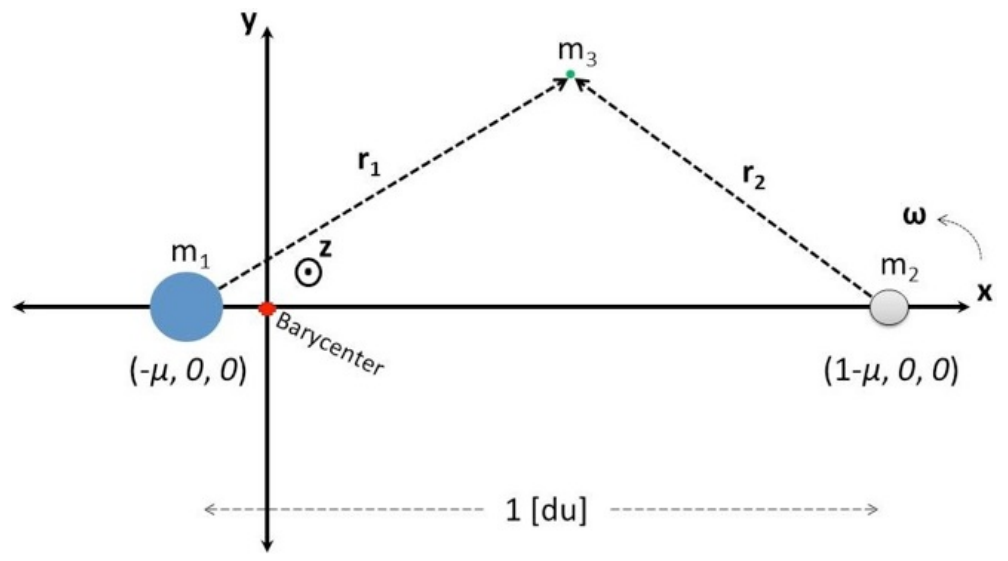

Figure 1.1: Basic setup for the CR3BP

The derivation for the equations of motion in the rotating synodic frame (the frame fixed relative to the primary and the secondary) is fairly straight-forward and well known, and it yields the following set of equations. See Vallado [11] for more details.

$$
\begin{gathered}
\ddot{x}=2 \dot{y}+x-\frac{\left(1-\mu^{*}\right)}{r_{1}^{3}}\left(x-\mu^{*}\right)-\frac{\mu^{*}}{r_{2}^{3}}\left(x+1-\mu^{*}\right) \\
\ddot{y}=-2 \dot{x}+y-\frac{\left(1-\mu^{*}\right)}{r_{1}^{3}} y-\frac{\mu^{*}}{r_{2}^{3}} y \\
\ddot{z}=-\frac{\left(1-\mu^{*}\right)}{r_{1}^{3}} z-\frac{\mu^{*}}{r_{2}^{3}} z
\end{gathered}
$$


Combining these equations and integrating the result yields the equation for Jacobi's Constant, $C$, a value somewhat akin to gravitational potential when the spacecraft has zero velocity relative to the synodic frame of the given system.

$$
C=x^{2}+y^{2}+2\left(\frac{\left(1-\mu^{*}\right)}{r_{1}}+\frac{\mu^{*}}{r_{2}}\right)
$$

These two sets of equations can applied to great effect. The non-intuitive nature of modeling motion that includes the effects of a second large body makes it incredibly difficult to imagine how orbits might have complex trajectile paths that are stable or even quasi-stable. These equations make it much easier to see how, relative to the rotating frame, orbits that utilize the geometry of the 2 large bodies in their shared space form intricate and repeating orbits. The Jacobi constant is also a very useful tool for thinking about the potential of certain orbits and their various and unique applications. It can be shown, by setting the relative velocity and acceleration to zero in the equations of motion, that there are specific locations that act as stable locations relative to the synodic frame. Stated otherwise, if the spacecraft could get to those points with infinite precision, and no perturbations acted upon the system, the spacecraft would stay in that location forever. It has been shown that these Lagrange points (Fig. 1.2) move in true $\mathrm{N}$ body simulations, but on a general conceptual level, it is reasonable to think of them as fixed. The figure below maps out the Jacobi energy in the Earth-Moon zero velocity space - the plot on the left shows the two dimension contours with the Lagrange points marked out with " $x$ "s, and the plot on the right shows the contours as a three dimensional surface. The plot on the right shows the negative Jacobi energy because it is more intuitive to think of the contours as gravity wells. The collinear $L_{1}, L_{2}$, and $L_{3}$ Lagrange points are saddle points, making them inherently unstable and forcing orbits around them to diverge easily without perfect conditions or continuous correction. The SOHO Mission, for example, made orbital maneuvers once every 20 days at the most frequent and 146 days at the most infrequent. These maneuvers used between .04 and $1.9 \frac{\mathrm{m}}{\mathrm{s}}$ for each 

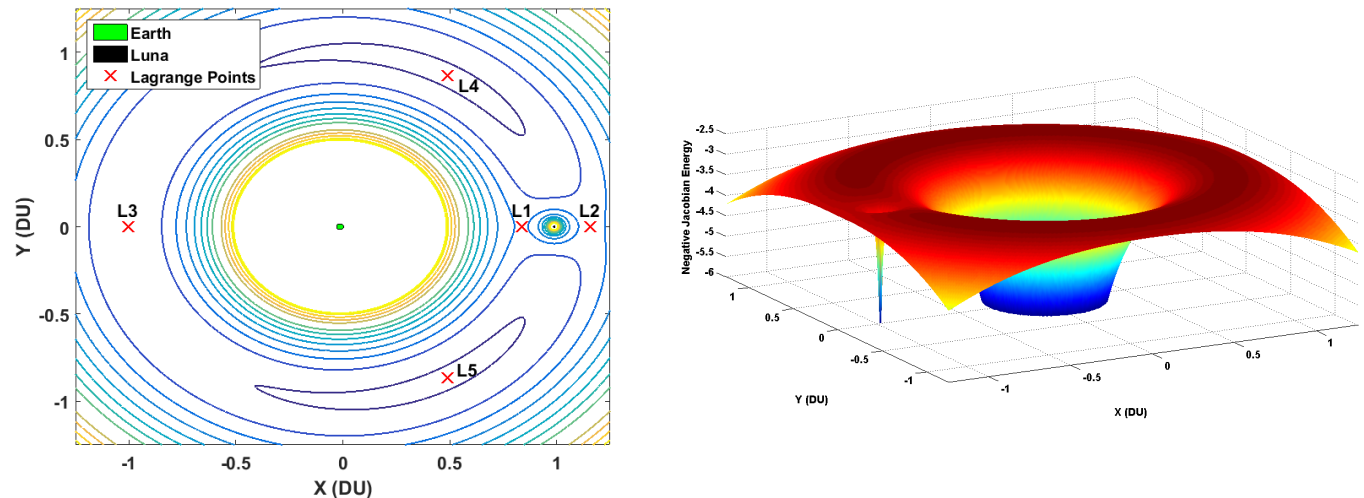

Figure 1.2: Energy curves and Lagrange points in the Earth-Moon CR3BP

maneuver [9]. The $L_{4}$ and $L_{5}$ points, on the other hand, are valleys, making them very stable, though their positions make orbits around them less interesting for potential missions. They also lose the advantage of being near the Moon that the $L_{1}$ and $L_{2}$ points have.

Richardson showed in his "Analytic Construction of Periodic Orbits About the Collinear Points" [8] that it was possible to form a third order solution for stable orbits around the first three Lagrange points in a three body system (Fig. 1.3). Halo orbits, as they're called due to the fact that they appear as though they are going in a halo around the moon from the perspective of an Earth observer, exhibit symmetry in the $\mathrm{y}-\mathrm{z}$ plane and the $\mathrm{x}-\mathrm{y}$ plane, but not in the $\mathrm{x}-\mathrm{z}$ plane due to the irregularities in equations of motion when in-plane with the two major bodies of system. The linearized equations of motion of a satellite in a halo orbit around one of the collinear Lagrange points are given in Richardson[8] as:

$$
\begin{gathered}
x^{*}=-A_{x} \cos (\lambda t+\phi) \\
y^{*}=k A_{x} \sin (\lambda t+\phi) \\
z^{*}=A_{z} \sin (\nu t+\psi)
\end{gathered}
$$

where the superscript indicates that the positions are relative to the chosen Lagrange 
point, not to the barycenter of the system. $A_{x}$ and $A_{z}$ represent the x-excursion and the z-excursion. Each represents the maximum distance from the geometric center of the orbit, which is close to the maximum distance that the satellite travels from the Lagrange point, though halo orbits tend to have their geometric center misaligned with the $\mathrm{x}-\mathrm{y}$ plane. Halo orbits whose centers are above the $\mathrm{x}-\mathrm{y}$ plane are part of the "northern" class of halos, and those below, the "southern". The $\lambda$ is the in-plane frequency of the orbit, $\nu$ the out-of-plane frequency, and $\phi$ and $\psi$ are phase offsets that don't actually change the shape of the orbit[8]. These equations are simple sinusoids and must be analyzed further in order to guarantee that they do not violate the CR3BP dynamics. Solving this problem and actually finding the relationships required to call the result in the given form a halo orbit is done by setting $\nu=\lambda$, making the in-plane and out-pf-plane frequencies the same. The solution is complex and not worth hashing out here. Figure 1.3 shows the result of the Richardson's third body approximations - a family of $L_{2}$ halo orbits. The halos in the figure represent a span of possible x-excursions, z-excursions, and both northern and southern classes.

Halos are the basis of orbital manifolds. Manifolds are projections of the possible trajectories on which a satellite will naturally tend towards or away from halos. This means that it only takes a very small perturbation to enter or exit from halo orbits to make what turn out to be very large transfers. To create manifolds from these equations, the first step is to find the Jacobian of the equations of motion at the Lagrange point in question. The eigenvectors of the Jacobian associated with its real eigenvalues give the direction of the perturbation required to produce manifolds from a given halo orbit - the larger eigenvalue and its paired eigenvector correspond to unstable manifolds, the smaller to stable manifolds. Unstable manifolds must be propagated forward in time and show the natural divergence of a body from a given halo while stable manifolds, propagated backwards in time, give families of trajectories that tend to converge on a given halo orbit (see Fig. 1.4). Both are important in 
planning low energy orbital transfers for Lunar and interplanetary missions.
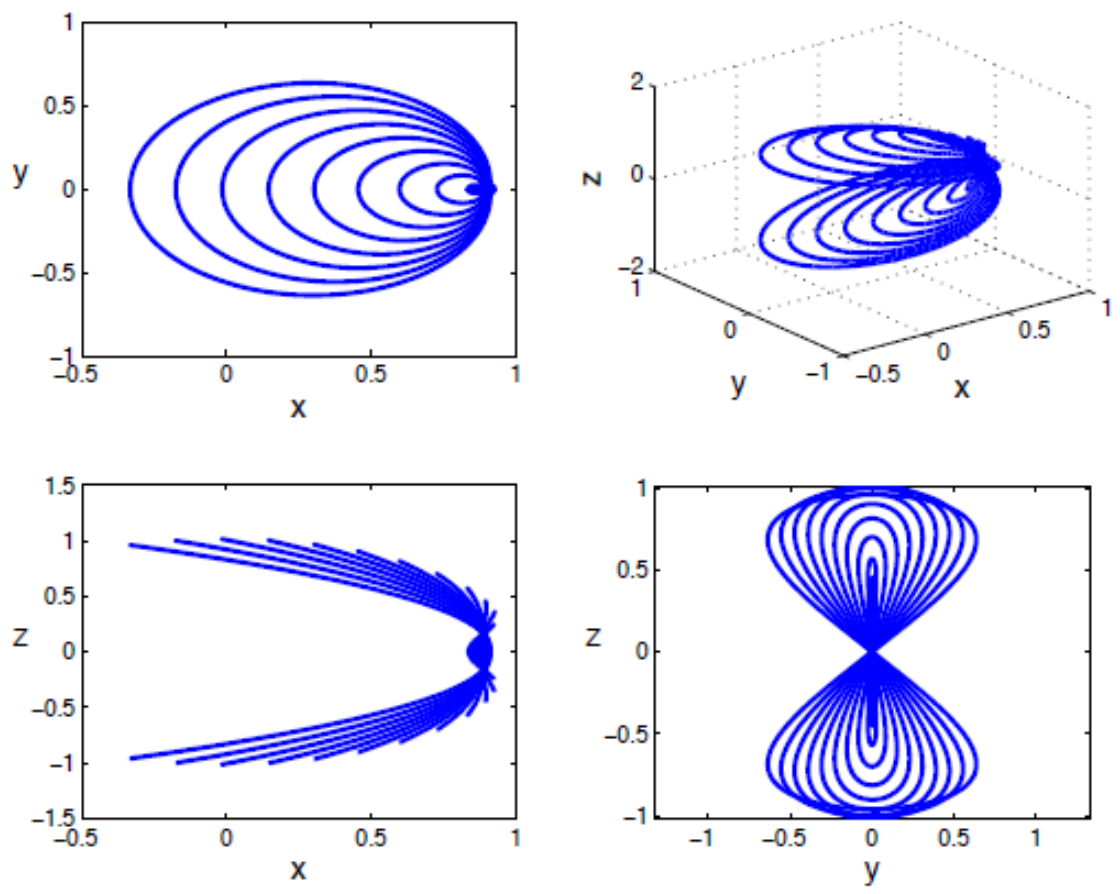

Figure 1.3: A Family of Lissajous Halo Orbits

These linearized equations can also be exploited to make families repeating orbits where the in-plane and out-of-plane frequencies are not the same. Archambeau, Augros, and Trelat [1], for example, exploit this to create families of 8-shaped Lissajous orbits with unique Lunar coverage properties and their own sets of invariant manifolds. The periodic and stability properties of halo orbits are excellent for missions that require a spacecraft to be more or less fixed relative to a certain position. The Earth-Moon $L_{1}$ point, the Lagrange point directly between the Earth and the Moon, has been used for certain observation missions in the past and the Earth-Sun $L_{1}$ point is the object of a number of different studies right now such as the James Web Space Telescope.

Manifolds are also the basis for a type of orbital transfer called a low energy 

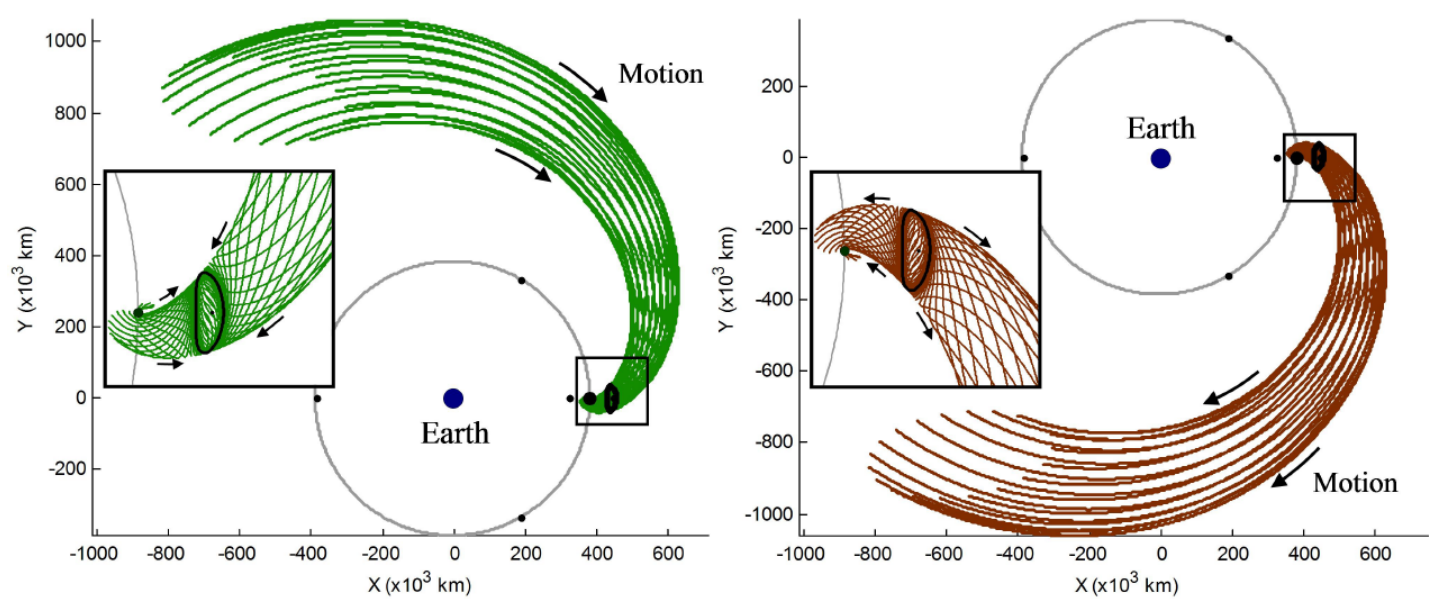

Figure 1.4: Stable (left) and Unstable (right) manifolds

transfer. Low energy transfers combine Earth-Sun stable manifolds that skim the surface of the Earth and then associated unstable manifolds that travel from the Earth-Sun $L_{1}$ (or $L_{2}$ ) to Earth-Moon manifolds. This allows bodies to travel from the Earth to the moon from nothing more than a launch trajectory. This capability has been demonstrated with such accuracy that a body was sent to the moon with a low-energy trajectory and returned to Earth, impacting a small area in a Utah test range. Other missions utilizing low energy trajectories range from the Apollo missions to the Surveyor missions and many more (??). In systems with lots of large bodies, the gas giant systems or the solar system, for example, low energy trajectories open up the possibility of "highways" between different planets. These highways would consist of a series of different manifolds that would allow a spacecraft to travel from one body to the next with little to no fuel continuously. This could mean that, potentially, the proper application of manifolds and low energy trajectories could produce a path that travels to every planet in the solar system and back again with almost no fuel requirements. The practicality is questionable due to the time scales this would require and what would most likely be extremely precise initial conditions, 
but the possibility is exciting nonetheless.

Manifolds also open the door to recurrence plots and Poincare maps, mathematical methods of studying phase spaces and trajectories as they relate to their "periodicity". Poincare maps allow a trajectory to be analyzed relative to how close different states of that trajectory are to one another in phase space. Stated otherwise, this mathematical technique can be used to create complex periodic orbit in sensitive dynamic systems, such as those in the CR3BP.

Applying the circular restricted three body problem to $\mathrm{N}$ bodies is not something that has been done in the same way that it is being explored in this thesis. There have been many papers published that have used a technique similar to patched conics in $\mathrm{N}$ body systems where individual three body systems are considered depending on which moon is closest to the satellite. This method seems viable for many systems, but it doesn't account for times when the satellite is close to multiple moons at the same time. The solutions created in this thesis attempt to address that problem by solving the circular restricted $\mathrm{N}$ body problem in the same way that the circular restricted 3 body problem addresses some of the issues that arise from patched conics near the borders of spheres of influence By always considering the effects of every body that is in the system, motion near the edge of influence of each body could be more accurately modeled. It also avoids the need for most arbitrary switching functions.

The first step, then, is to prove that solving is the CRNBP is even possible. Once a basis of equations is established, the next step is to test the results to see if they are sensible. One proven to be feasible, these systems need to be applied to a real-world system and tested to see if they can produce the interesting and useful trajectories produced in the CR3BP. By showing that the circular restricted $\mathrm{N}$ body problem can be used in more than heuristic ways, the model can then gain viability and possible 
lend itself to the type of fast, complex trajectile analysis that is so pragmatic, easily accessible, and low cost. Such is the purpose of this thesis.

The trajectories of planet-bound satellites can be modeled, with closed solutions for Jacobian energy and the equations of motion, in a similar fashion to the CR3BP for $\mathrm{N}$ bodies given the additional assumption that the barycenter of the planetary system is near the location of the planet itself. This is valid for any system where the planets mass is significantly larger than that of its largest satellites. The solution to this CRNBP can be used to simulate and plan low energy transfers in systems with more than 3 bodies and it can potentially be used to more accurately plan trajectories in the Jovian system compared to the traditional method of coupling 3 body systems, with no notable increase in computational time.

This thesis will be structured as follows. First it will be discussed how the equations of motion are derived and modeled in the CRNBP as well as the equations for the Jacobi energy. Both of these will be verified with test cases. Then these equations will be applied to the Jovian system to look at the existence and possibility of stable points like Lagrange points, and periodic orbits. After that, halo orbits will be modeled and analyzed - one method for correcting and maintaining these orbits will be introduced as well. Finally, the thesis will look at the possibility of using CRNBP dynamics to model low energy transfers in the Jovian system. 
Chapter 2

\section{THE CIRCULAR RESTRICTED N BODY PROBLEM}

\subsection{Derivation of the Equations of Motion}

The purpose of this first section is to establish the equations of motion of a small body in an $\mathrm{N}$ body system. The results are then verified in the proceeding subsection.

The N-body system is initially constructed in an almost identical fashion to the 3-body problem with the second largest mass being situated 1 distance unit from the largest body along the $\mathrm{x}$-axis of the synodic frame of the system. The setup is given in Figure 2.1. The important differences to note in the setup are that the barycenter of the system is considered to be at the center of the largest body - why this is assumption is made becomes apparent later on - and the mass ratio is now based on a mass unit equal to the sum of all of the gravitationally relevant masses. The system's synodic frame is relative to the largest two bodies, and, as before, the major bodies are assumed to be coplanar and moving in circular orbits.

The motion of the satellite around the primary can be modeled using standard equations of gravitation including the perturbing effect of the secondaries. For the

rest of the discussion in this paper, the "primary" is the largest body, the planet, and the "secondaries" are all of the other large bodies in the system.

$$
\ddot{\vec{r}}_{1 s}=-\sum_{i=1}^{N} \mu_{i} \frac{\vec{r}_{i s}}{r_{i s}^{3}}-\sum_{j=2}^{N} \mu_{j} \frac{\vec{r}_{1 j}}{r_{1 j}^{3}}
$$

If $\mu_{i}$ is the gravitational constant of the $i^{\text {th }}$ body with respect to the satellite, i.e. $\mu_{2}=G\left(m_{2}+m_{s}\right) \approx G m_{2}$, the first summation represents the direct effect, or the force cause by each body pulling on the satellite itself, and the second summation, the indirect effect, represents the force caused by each secondary body pulling on the 


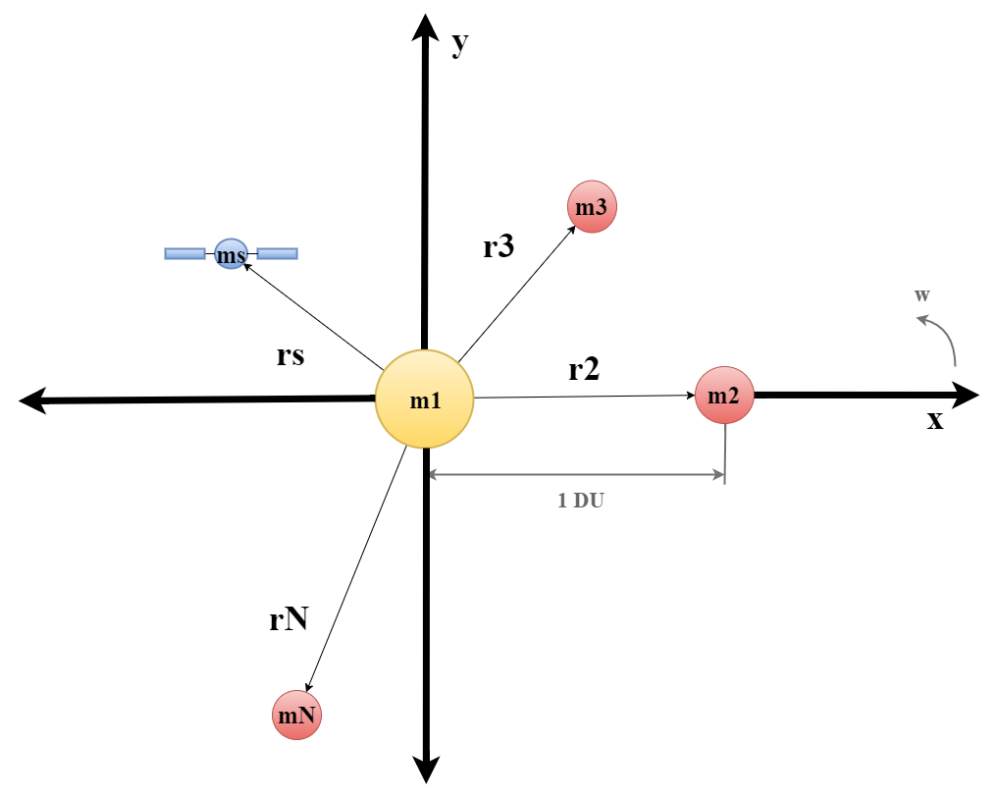

Figure 2.1: Basic setup for the CRNBP

primary pulling on the satellite. Basic equations of motion for a body in a synodic frame give the following formula by comparison:

$$
\ddot{\vec{r}}_{1 s}=\ddot{\vec{r}}_{B s}-\omega_{S y n}^{2}\left(x_{s a t} \hat{x}_{S y n}+y_{s a t} \hat{y}_{S y n}\right)-2 \omega_{S y n}\left(\dot{y}_{s a t} \hat{x}_{S y n}+\dot{x}_{s a t} \hat{y}_{S y n}\right)
$$

From here on out, the subscript $s$ will be used in place of sat and it will be assumed that each directional component of any vector is defined relative to the synodic frame unless otherwise stated. The rate of rotation of the frame can be simplified by scaling it to equal one. Setting these two equations equal to each other, solving for the acceleration of the satellite with respect to the inertial frame, and breaking the result into it's respective components, the equations of motion resemble those from the CR3BP but with a small perturbational component from the indirect effect:

$$
\begin{gathered}
\ddot{x}_{s}-2 \dot{y}_{s}-x_{s}=-\sum_{i=1}^{N} \mu_{i} \frac{x_{s}-x_{i}}{r_{i s}^{3}}-\sum_{j=2}^{N} \mu_{j} \frac{x_{j}}{r_{1 j}^{3}} \\
\ddot{y}_{s}+2 \dot{x}_{s}-y_{s}=-\sum_{i=1}^{N} \mu_{i} \frac{y_{s}-y_{i}}{r_{i s}^{3}}-\sum_{j=2}^{N} \mu_{j} \frac{y_{j}}{r_{1 j}^{3}}
\end{gathered}
$$




$$
\ddot{z}_{s}=-\sum_{i=1}^{N} \mu_{i} \frac{z_{s}}{r_{i s}^{3}}
$$

The fact that the bodies are being treated as coplanar eliminates any perturbations in

the $\hat{z}$ direction, making the $\ddot{z}_{s}$ identical to that of the CR3BP. For $\hat{x}$ and $\hat{y}$, however, the effects of the secondaries pulling on the primary creates an $\frac{1}{r^{2}}$ decaying effect with respect to the secondary's distance from the primary, exactly what you would expect. If the secondary is closer, it pulls the primary more, and thus perturbs the satellite likewise. None of this, however, is exceptional or new in any respect. The equations of motion for a small body orbiting a large one has been modeled including the n-body gravitational effects at great length. Then only difference here is the combination of these well known equations of motion (the right hand side of ??, ??, and ??) with the motion of a body relative to a rotating frame.

\subsubsection{Test Cases}

To verify these equations of motion, they were simulated using Matlab's ode45 function and compared against simulation of the equations of motion for the 3 body problem. Both three and two dimensional simulations were run with similar outcomes so for the sake of simplicity, only two dimensional cases are shown here. Since the only difference between the two sets of equations is a perturbing force in the $\mathrm{x}-\mathrm{y}$ plane, z motion will be identical given the same starting conditions. Both of the plots in Fig. 2.2 demonstrate the difference between the EOM for the CR3BP and the CRNBP. Cases where the indirect effect is minimized, orbits that stay close to Earth or simply far from the moon, tend to exhibit almost identical behavior (left). When the orbit tends closer to the moon, however, a notable difference becomes apparent, though both systems tend towards similarly quasi-periodic, sinusoidal trends (right). Even when the initial position is pushed closer and closer to the L1 point, both spacecraft travel in similar paths. There is only a very small range that causes the two 

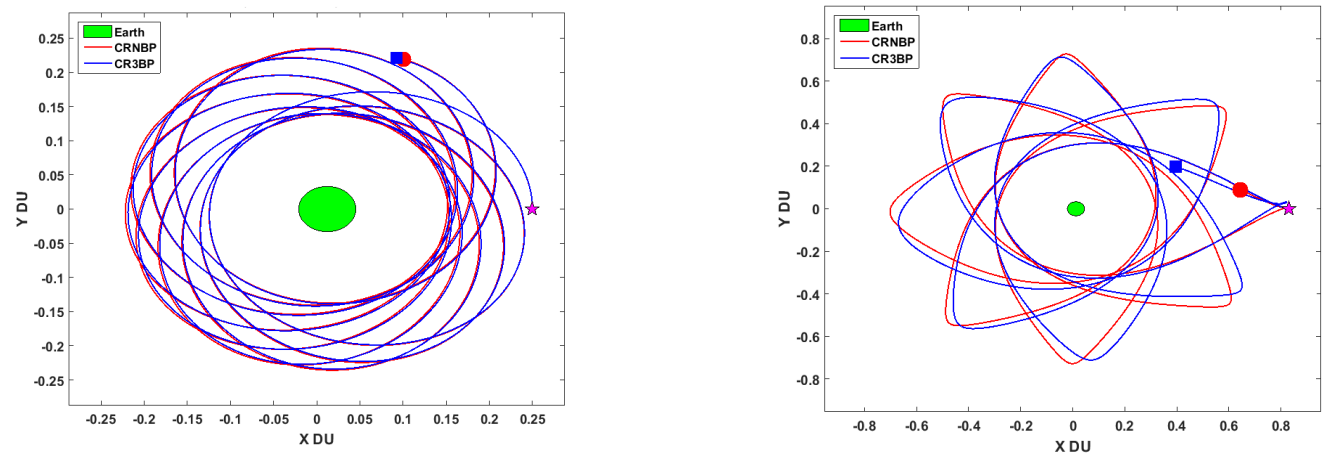

Figure 2.2: Trajectory comparisons between the CRNBP (red) and the CR3BP (blue). Both are given the same starting conditions

types of path to diverge, but, despite this, the similarities between the systems are still obvious.

A spacecraft is limited to how far it can get away from the Earth in a way that is related to the Jacobi constant - i.e. when a spacecraft has a given launch energy, that energy corresponds to some Jacobi value that the satellite cannot fly past without some change in momentum. Figure 2.3 shows this by overlaying the energy curves of the system on top of the satellites trajectory. The perturbations caused by the
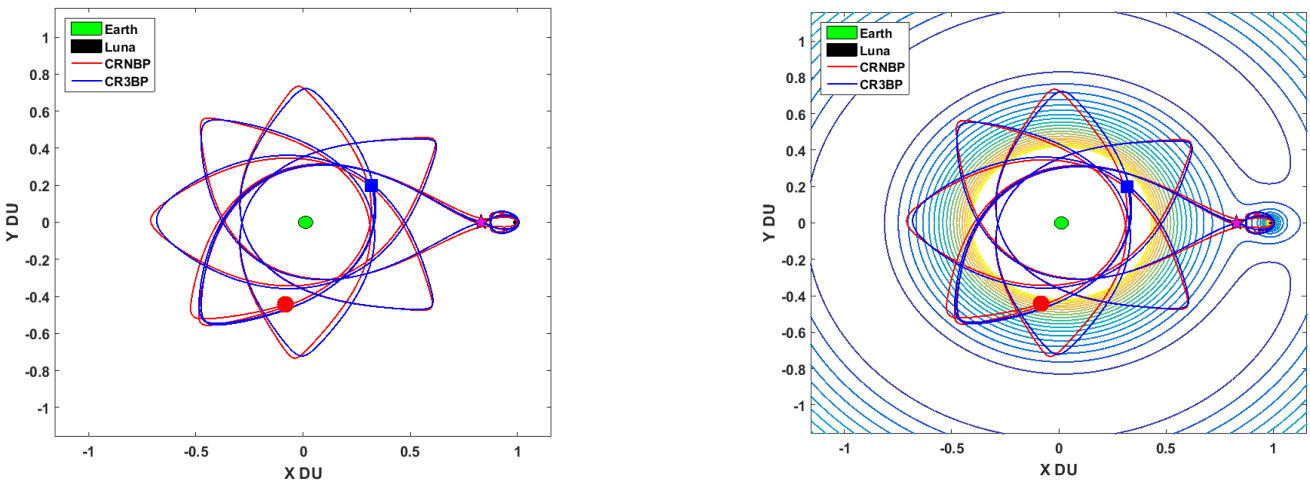

Figure 2.3: A test trajectory with quasi-stable trans-lunar orbiting properties. The plot on the left has the energy curves of the system laid over top of it

moon pulling on Earth, assuming the Earth were at the barycenter of the Earth- 
Moon system, would make the force of gravity felt by the spacecraft less when it was eclipsed to the moon. We know that this is an assumption, however, and for a system like the Earth-Moon system where the Earth is displaced from the barycenter by the mass ratio of the moon, it is more inaccurate. These merely act as a sanity check. Looking at systems where the mass of the primary is even more dominant, the assumption of placing the primary at the barycenter becomes less and less inaccurate, as do the results of these equations. A study should be conducted to see at what point the mass ratio becomes too large for this assumption to be valid. For the scope of this thesis, the main focus being the Jovian system and the Solar system, the mass ratios are exceedingly high - Jupiter accounts for approximately $99.98 \%$ of the total mass of it and its four largest moons - Ganymede, the second largest moon in the system weighs in at less than $.008 \%$. By comparison, the Earth makes up about $98.78 \%$ of the Earth-Moon system, the Moon about 1.22\%. This means that, even if the top four most massive secondary bodies in the Jovian system were to be smashed into one large mass, that mass would still have to increase by two orders of magnitude in order to have the same effect that Luna does on Earth.

There was also an important underlying assumption that was added to the CRNBP that requires verification before moving to a point where the results of this thesis could even be accepted as academically relevant if not pragmatic. The assumption that the barycenter of the system is at the center of the largest body needs to be verified as a valid assumption. It is possible with the delicacy of orbits in these systems that even moving the barycenter by a fraction of a percentage could have dramatic effects on both specific trajectories and path trends. Halo orbits, for example, require extremely high precision to create, and are so sensitive in reality that is impossible to maintain them for long periods without some kind of correction. Naturally, even without the barycenter moving, the extra bodies in the CRNBP would cause divergence. It might even be possible for the adverse dynamics caused by the motion of the barycenter 
would cause entirely different types of orbits. It is therefore imperative to check that this assumption does not render the results of this thesis functionally useless.

The most straight forward method for testing the validity of this assumption was to run a simulation wherein this assumption was not made and each body, including the primary, was propagated according to traditional Newtonian gravitational dynamics. Though this method is simple enough conceptually, being nothing more than a vector sum for the force acting upon each body by all other bodies, it is computationally expensive and it is weakly chaotic over long periods of time. To mitigate these issues, comparative simulations were limited in scope and were meant to show that both systems, the high fidelity simulation without outside perturbations and the CRNBP, had similar trends given similar initial conditions. Though there might be noticeable differences in the final state of a body propagated through each system, what is important for this thesis is the way that the trajectories trend. The goal of solving these types of problems, for the scope of this thesis, is to validate their potential as a viable modeling system and to explore the possible types of trajectories that are valid while the perturbations caused by other large bodies are being considered.

All of the results for these comparison have the same format as the comparisons made before between the CRNBP and the CR3BP in the Earth-Moon system where the star indicates the start point of both simulations and the square and the circle represent the end points of the high precision propagation (HPP) and the CRNBP respectively. Unless otherwise stated, all the figures show 1 month long simulations. In most cases, the two cases were almost entirely identical for a reasonable time span, and the ones that tended to diverge only did so due to strange interactions. Figure 2.4 gives two examples of the former, where both cases stayed together for the entire simulation with very minor differences on the scale of the problem. For example, the final difference between the two propagated bodies in the figure on the right is 96,000 $\mathrm{km}$ and the $40,000 \mathrm{~km}$ on the left. Although this seems like a large distance, it is due 
to the magnitude of the distances in the propagation. Each of these distances only represents .09\% and .04\% of the distance between Jupiter and Ganymede respectively. Figure 2.5, on the contrary, is a case of the latter, where the two cases did not stay
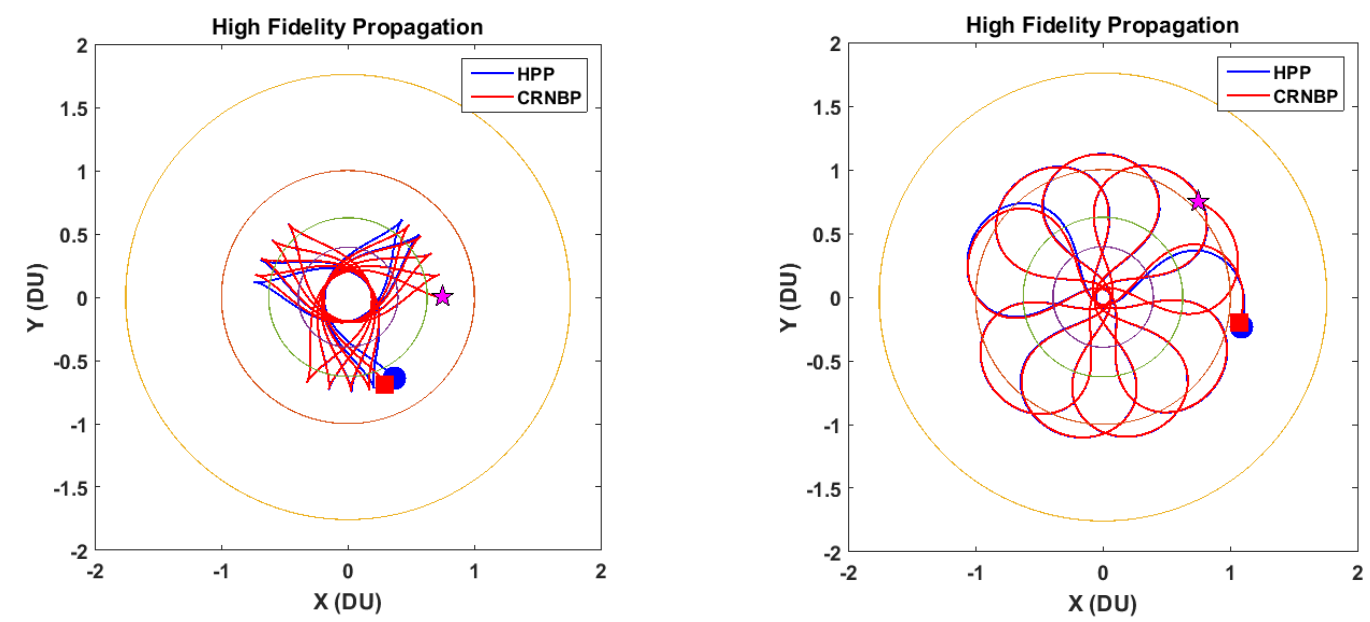

Figure 2.4: Comparison to High Fidelity Propagation

together. The tendency of the two cases to diverge from one another in short time spans was dictated by whether or not the satellite flew too close to one of the planets. In this example the black circle identifies the point of divergence before which the two propagations had stayed together. This occurred because the satellite flew either extremely close to Ganymede or entirely through it in one of the simulations. For any trajectories that had very close interactions with the planet or any of the moons, it seems that the small differences in the locations of the larger bodies due to their assumed motion did end up having a large impact on the final state of the simulation. The motion of bodies in the system, however, did trend similarly, which is important within the scope of this paper. It is also important to gauge the validity that the center of the largest planet lines up with the barycenter of the system more directly. To do so, an orbit is chosen that doesn't appear to have any major interactions with the planet or any of the moons. This orbit is then pushed out in time until the two cases, the HPP and CRNBP cases, diverge significantly. The orbit chosen is shown 


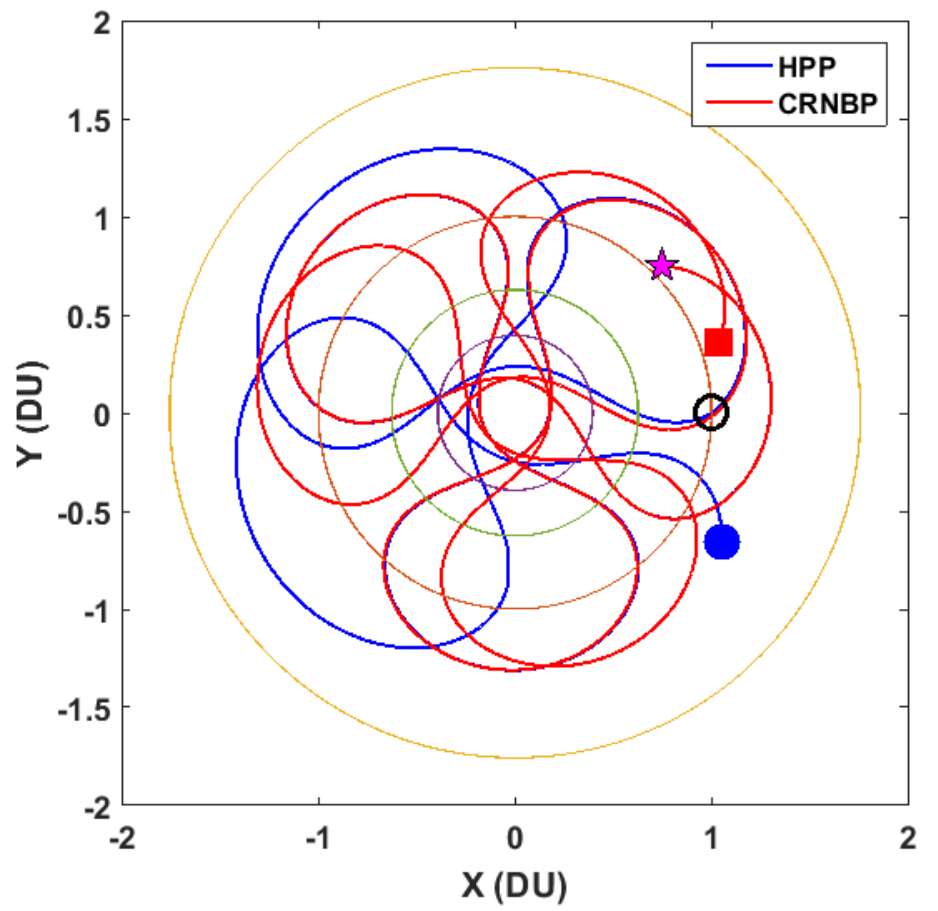

Figure 2.5: Divergent Comparison to High Fidelity Propagation

on the left in Fig. 2.6, propagated out for 1 month, and again on the right for 2 months. It is evident that neither of these cases show any significant deviation for
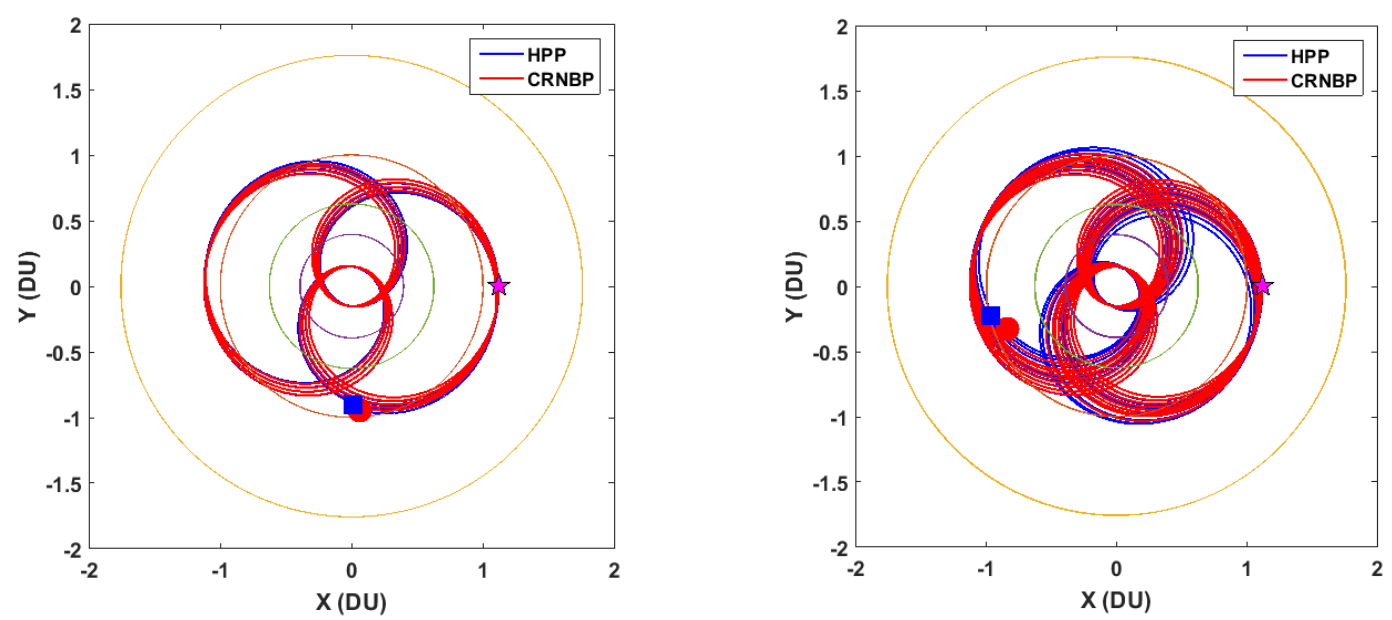

Figure 2.6: Long Duration Comparison to High Fidelity Propagation

one another despite the relatively large time span. To test the assumption further, 
this same orbit was tested in each system with the mass ratio of the Galilean moons increased above its current value of $0.0207 \%$. By incrementally increasing the mass ratio of the moons to the planet, it should become clear at what point systems like the Jovian system can no longer be accurately modeled using these assumptions.

Since we have already tested these equations in the Earth-Moon system, the mass ratio was initially increased by an order of magnitude, putting it still well below the Earth-Moon mass ratio of $1.22 \%$. The results for tests for a mass ratio of $0.04 \%$ and $0.1 \%$ (Figure 2.7), and $0.2 \%$ and $2 \%$ (Figure 2.8) are shown below. The first
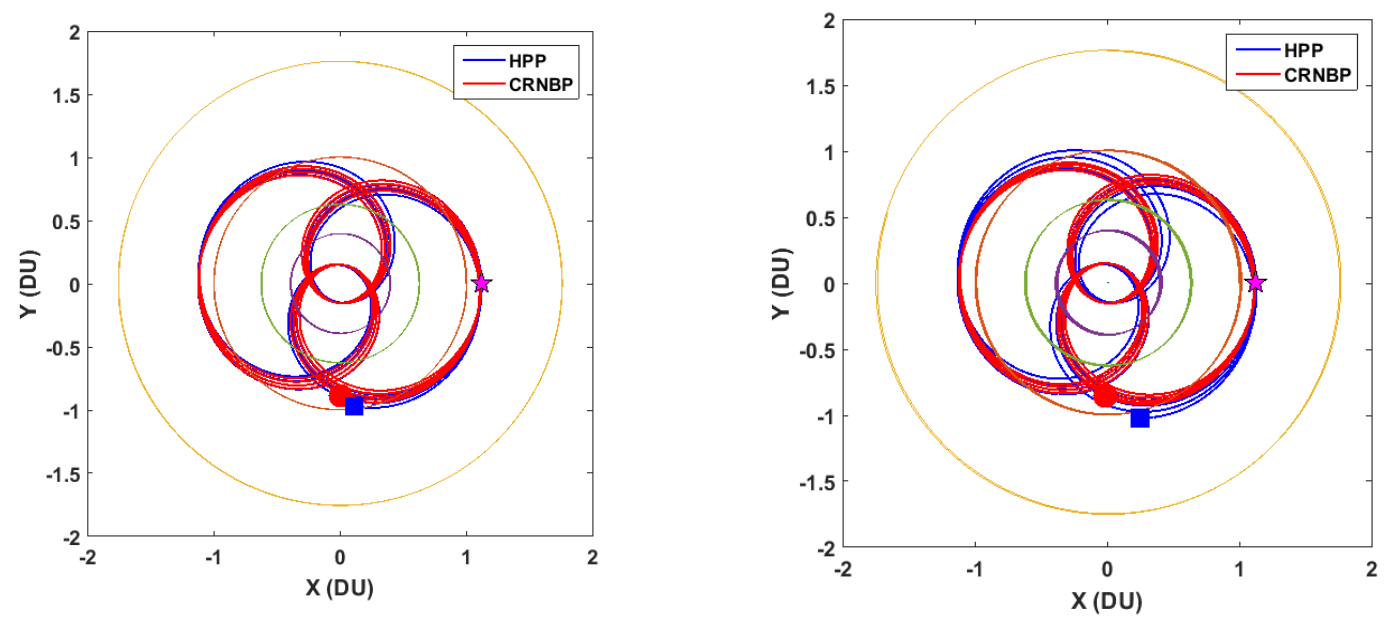

Figure 2.7: Mass Ratio Tests (x2 and x5)

set of tests show little difference in the results of the two propagations. Even with a mass ratio of $0.1 \%$, the difference is minimal and the trends of the orbits remain the same. We do notice, however, a slight elongation of the eccentricity of the high precision propagation that is exaggerated by pushing the mass ratio up to $0.2 \%$. At this mass ratio, it is also evident that the motion of the moons is no longer circular, though it is probably still accurate to say that it behaves similarly to circular orbits. The slight oscillation of the moons seems to be what is causing the oscillation in the trajectory of the HPP. The comparison between trajectories when the mass ratio is around $2 \%$ becomes a reductio ad absurdum to the assumption that that the mass 

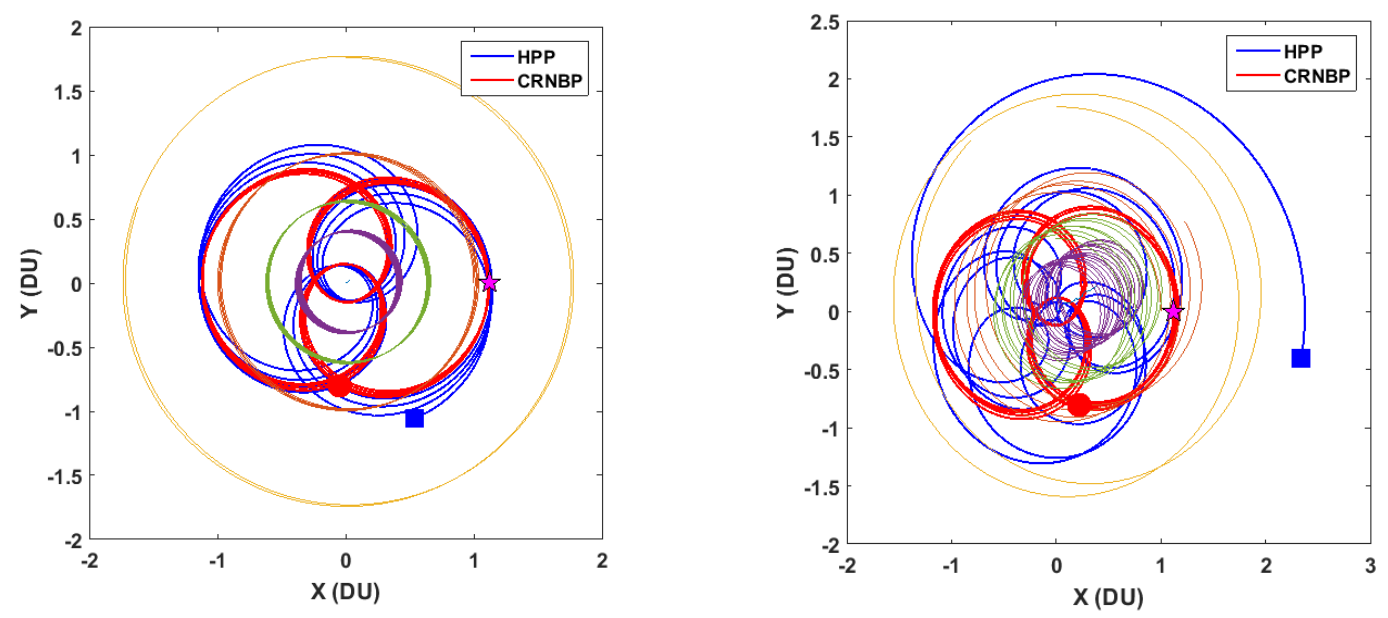

Figure 2.8: Mass Ratio Tests (x10 and x100)

ratios of the system do not make a large difference in the motion of the satellite. At this point, which is actually fairly close to the mass ratio of the Earth-Moon system, the Jovian system breaks down. Not only do the orbits not resemble anything like circular motion, but the motion of the satellite is wild and unpredictable, certainly nothing like that from the CRNBP.

The results of this verification are ambiguous for a few reasons. On one hand, it seems like there are always cases in which the trajectory of the CRNBP can not be accurately modeled, especially in cases where the satellite comes very close to any of the major bodies. On the other hand, it seems within the scope of reason to imagine that, so long as statements about the assurance of the existence of these trajectories as shown are avoided, the CRNBP has both the form and function of high fidelity propagation methods without the computationally expensive requirements. For the Jovian system, this is almost certainly the case. Once the mass ratio of the Jovian system reaches something like 1\%, perhaps even lower, the dynamics break down and the model no longer functions, but for the majority of cases with the actual mass ratio of the system, the CRNBP seems to correctly model the trends of orbits. A more careful look at exactly where this mass ratio breaks apart the assumptions of 
the system would be required to verify the accuracy of the results further.

\subsection{The Jacobi Constant}

The purpose of this section is to establish the equations for the Jacobi constant in

the N-body problem. The results are straightforward and appear consistent with the results from the 3-body system. To derive the Jacobi Constant of the associated system, a similar solution process to that of the CR3BP is required once again. Each equation, ??, ??, and ??, is multiplied by $2 \dot{x}_{s}, 2 \dot{y}_{s}$, and $2 \dot{z}_{s}$ respectively.

$$
\begin{gathered}
2 \dot{x}_{s} *\left[\ddot{x}_{s}-2 \dot{y}_{s}-x_{s}=-\sum_{i=1}^{N} \mu_{i} \frac{x_{s}-x_{i}}{r_{i s}^{3}}-\sum_{j=2}^{N} \mu_{j} \frac{x_{j}}{r_{1 j}^{3}}\right] \\
2 \dot{y}_{s} *\left[\ddot{y}_{s}+2 \dot{x}_{s}-y_{s}=-\sum_{i=1}^{N} \mu_{i} \frac{y_{s}-y_{i}}{r_{i s}^{3}}-\sum_{j=2}^{N} \mu_{j} \frac{y_{j}}{r_{1 j}^{3}}\right] \\
2 \dot{z}_{s} *\left[\ddot{z}_{s}=-\sum_{i=1}^{N} \mu_{i} \frac{z_{s}}{r_{i s}^{3}}\right]
\end{gathered}
$$

Combining theses yields:

$$
\begin{gathered}
L H S=2\left(\dot{x}_{s} \ddot{x}_{s}+\dot{y}_{s} \ddot{y}_{s}+\dot{z}_{s} \ddot{z}_{s}\right)-2\left(x_{s} \dot{x}_{s}+y_{s} \dot{y}_{s}\right) \\
R H S=-2\left(\sum_{i=1}^{N} \mu_{i} \frac{\left(x_{s}-x_{i}\right) \dot{x}_{s}+\left(y_{s}-y_{i}\right) \dot{y}_{s}+z_{s} \dot{z}_{s}}{r_{i s}^{3}}+\sum_{j=2}^{N} \mu_{j} \frac{x_{j} \dot{x}_{s}+y_{j} \dot{y}_{s}}{r_{1 j}^{3}}\right)
\end{gathered}
$$

Again, we haven't diverged much from the three body problem. The LHS is identical and the right hand side only has the second summation from the indirect effect to differentiate it. Integrating equation ?? is straightforward and uninteresting but equation ?? requires a little more work.

$$
\int L H S=\int 2\left(\dot{x}_{s} \ddot{x}_{s}+\dot{y}_{s} \ddot{y}_{s}+\dot{z}_{s} \ddot{z}_{s}\right)-2\left(x_{s} \dot{x}_{s}+y_{s} \dot{y}_{s}\right)=\dot{x}_{s}^{2}+\dot{y}_{s}^{2}+\dot{z}_{s}^{2}-\left(x_{s}^{2}+y_{s}^{2}\right)
$$




$$
\int R H S=-2\left(\int \sum_{i=1}^{N} \mu_{i} \frac{\left(x_{s}-x_{i}\right) \dot{x}_{s}+\left(y_{s}-y_{i}\right) \dot{y}_{s}+z_{s} \dot{z}_{s}}{\sqrt{\left(x_{s}-x_{i}\right)^{2}+\left(y_{s}-y_{i}\right)^{2}+z_{s}^{2}}}+\int \sum_{j=2}^{N} \mu_{j} \frac{x_{j} \dot{x}_{s}+y_{j} \dot{y}_{s}}{r_{1 j}^{3}}\right)
$$

If you notice that the derivative of the term inside of the square root in the denominator is the numerator, the first term in ?? becomes trivial to integrate. The second term, would, however, be impossible to integrate if not for the assumption that the primary is approximately at the barycenter of the given system. This means the $r_{1 j}$ can be treated as a constant and pulled out of the integral.

$$
\int \sum_{j=2}^{N} \mu_{j} \frac{x_{j} \dot{x}_{s}+y_{j} \dot{y}_{s}}{r_{1 j}^{3}}=\sum_{j=2}^{N} \frac{\mu_{j}}{r_{1 j}^{3}} \int x_{j} \dot{x}_{s}+y_{j} \dot{y}_{s}
$$

This is a little tricky to integrate in its current form. Converting the equation into polar coordinates and only considering one term at a time makes the problem more approachable.

$$
\int x_{j} \dot{x}_{s}=\int r_{j} \cos \left(\theta_{j}\right)\left[\frac{d}{d t}\left(r_{s} \cos \left(\theta_{s}\right)\right)\right]
$$

Simplifying this expression using separation by parts yields

$$
=r_{j} \cos \left(\theta_{j}\right) r_{s} \cos \left(\theta_{s}\right)-\int\left(\dot{r}_{j} \cos \left(\theta_{j}\right)-r_{j} \dot{\theta}_{j} \sin \left(\theta_{j}\right)\right) r_{s} \cos \left(\theta_{s}\right)
$$

Since we know that $\dot{r}_{j}$ is zero and, since we know that the bodies are in circular orbits, $\dot{\theta}_{j}$ therefore being constant, we can rearrange the equation into the following,

$$
=r_{j} \cos \left(\theta_{j}\right) r_{s} \cos \left(\theta_{s}\right)+\dot{\theta}_{j} \int\left(r_{j} \sin \left(\theta_{j}\right)\right) r_{s} \cos \left(\theta_{s}\right)
$$

Now we convert back into Cartesian coordinates.

$$
=x_{j} x_{s}+\dot{\theta}_{j} \int x_{s} y_{j}
$$

The solution to the integral with both $\mathrm{x}$ and $\mathrm{y}$ terms is then,

$$
\int x_{j} \dot{x}_{s}+y_{j} \dot{y}_{s}=x_{j} x_{s}+y_{j} y_{s}+\dot{\theta}_{j} \int x_{s} y_{j}-y_{s} x_{j}
$$


We can now focus on the remaining integral. Notice that the integral is equivalent to the $\vec{r}_{s} \times \vec{r}_{j}$, which can then be rewritten using the sine rule.

$$
\int x_{s} y_{j}-y_{s} x_{j}=\int \vec{r}_{s} \times \vec{r}_{j}=\int\left\|\vec{r}_{s}\right\|\left\|\vec{r}_{j}\right\| \sin \left(\theta_{s}-\theta_{j}\right)=r_{s} r_{j} \int \sin \left(\theta_{s}-\theta_{j}\right)
$$

Now we have something that is almost solvable. Since we know that $\theta_{j}$ is linear in time, our only concern would be integrating $\theta_{s}$. If we remember from the derivation of the CRNBP, the Jacobi constant is only concerned with the zero velocity values of C, we know that $\dot{x}_{s}=\dot{y}_{s}=\dot{z}_{s}=0$. If the velocity is zero, the rotational velocity, $\dot{\theta}_{s}$, is also zero, and $\theta_{s}$ is, therefore, a constant in time.

$$
r_{s} r_{j} \int \sin \left(\theta_{s}-\theta_{j}\right)=\left(r_{s} r_{j}\right)\left(\frac{1}{\dot{\theta}_{j}} \cos \left(\theta_{s}-\theta_{j}\right)\right)
$$

To simplify this, we can use the formula for angle subtraction and basic polarCartesian conversions.

$$
r_{s} r_{j} \cos \left(\theta_{s}-\theta_{j}\right)=r_{s} r_{j}\left(\cos \left(\theta_{s}\right) \cos \left(\theta_{j}\right)+\sin \left(\theta_{s}\right) \sin \left(\theta_{j}\right)\right)=x_{s} x_{j}+y_{s} y_{j}
$$

We also see that the $\dot{\theta}_{j}$ terms cancel out. Plugging this back into ?? yields a relatively simple result.

$$
\int x_{j} \dot{x}_{s}+y_{j} \dot{y}_{s}=2\left(x_{j} x_{s}+y_{j} y_{s}\right)
$$

The solution to equation ??, therefore, is given by:

$$
\int R H S=-2\left(\sum_{i=1}^{N} \mu_{i} \frac{1}{\sqrt{\left(x_{s}-x_{i}\right)^{2}+\left(y_{s}-y_{i}\right)^{2}+z_{s}^{2}}}+2 \sum_{j=2}^{N} \mu_{j} \frac{x_{j} x_{s}+y_{j} y_{s}}{r_{1 j}^{3}}\right)
$$

Combing equation ?? with ?? gives us the fully explicated integral of the equations of motion and the base equation for the Jacobi constant, $C$. We can also simply ?? by noting that $\dot{x}_{s}^{2}+\dot{y}_{s}^{2}+\dot{z}_{s}^{2}$ is the speed of the satellite squared, $V_{s}^{2}$.

$$
V_{s}^{2}-\left(x_{s}^{2}+y_{s}^{2}\right)=-2\left(\sum_{i=1}^{N} \mu_{i} \frac{1}{r_{i s}}+2 \sum_{j=2}^{N} \mu_{j} \frac{x_{j} x_{s}+y_{j} y_{s}}{r_{j}^{3}}\right)-C
$$


As said before, we are only concerned with the zero-velocity curves associated with the Jacobi constant, so $V_{s}$ is set to zero and equation ?? is rearranged to solve for $C$.

$$
C=x_{s}^{2}+y_{s}^{2}-2\left(\sum_{i=1}^{N} \frac{\mu_{i}}{r_{i s}}+2 \sum_{j=2}^{N} \mu_{j} \frac{x_{j} x_{s}+y_{j} y_{s}}{r_{1 j}^{3}}\right)
$$

Comparing this to equation ??, the equation for the Jacobi constant in the CR3BP, the only difference is the term that resulted from the inclusion of the indirect effect.

\subsubsection{Test Cases}

The purpose of this section is to verify the equations derived in the previous section and, since there is not much discussion of the Jacobi constant after this section, to briefly explore and analyze the Jacobi energy of the Jovian system. Although it might seem pertinent to compare the results of these equations to those of the CR3BP using a well known system like the Earth-Moon system, the results of analyzing the Jacobi constant of $\mathrm{N}$ body system are similar enough to the 3 body system, that the comparison is not necessary here.

To keep the system closer to the CR3BP and to make the results easier to analyze, the Galilean moons were all placed along the x-axis. As Fig. 2.9 shows, the energy curves of the Jovian system are reminiscent of the three body system, with the value spiking near each of the bodies, but the effects of the secondaries are much less prominent due to the overwhelming gravitational force from Jupiter. It is also evident that this system shares the same general trends as any three body system, with the largest valley in the energy curves occurring at the same radius as the largest secondary. Although it's difficult to make out, the interaction between Jupiter and Ganymede creates a stable region on the opposite side of the planet as Ganymede, right around where the $L_{3}$ point would be. When the moons aren't aligned, the different valleys move in accordance with the geometry of the system, but the size of the valleys relative to the system as a whole is unchanged even for different geometries. 

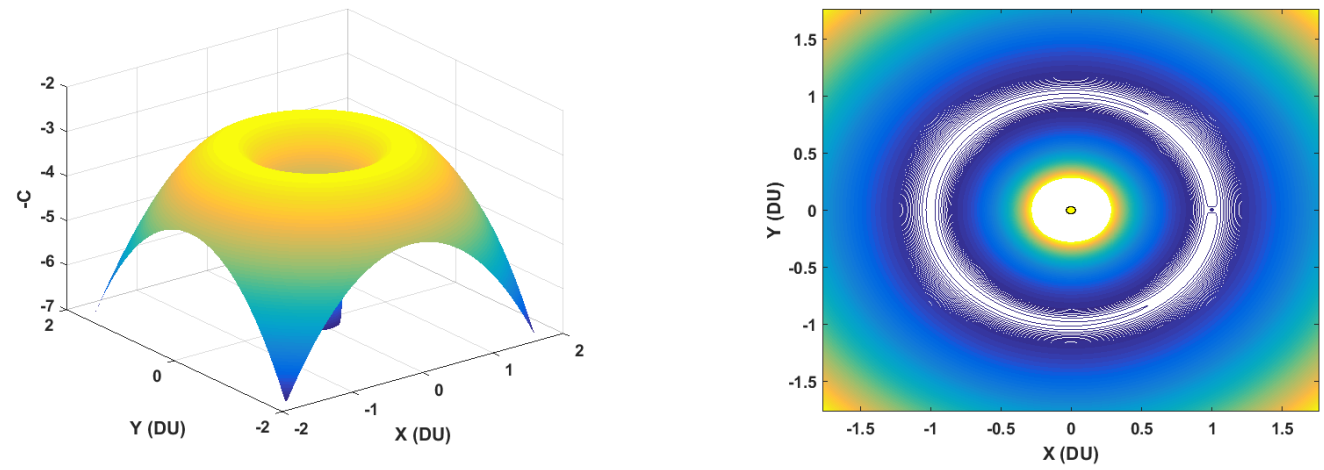

Figure 2.9: Two and three dimensional plots of the Jacobi energy for the Jovian system

Near Ganymede, the system trends similarly to the area near the Moon in the Earth-Moon system. The constant energy lines go from one side of the moon to the other, suggesting that trajectories that go from the Jupiter-Ganymede $L_{1}$ to the $L_{2}$ are possible even with the perturbations from the extra bodies. It is shown directly that this is possible later in this paper. The other moons, however, don't display the same type of behavior. The areas in proximity to each of the moons are displayed in
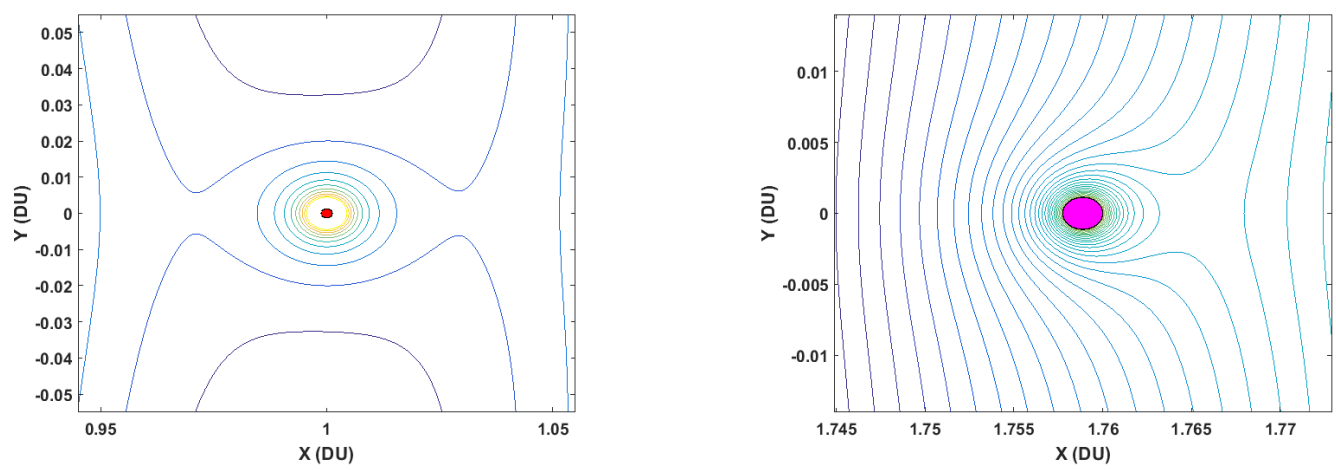

Figure 2.10: Jacobi Energy near Ganymede and Callisto

Figures 2.10 and 2.11. Unlike the area near Ganymede, where there symmetric level curves surrounding the body, the energy curves near Callisto, Io, and Europa seem to heavily favor one side. For the moons that are closer to Jupiter than Ganymede, there appears to more stable regions on the inside of the moon, while the opposite is try for 
moons outside of the radius of Ganymede. This behavior could be extrapolated from the dramatic slopes displayed in first figure, but the physical explanation for why this occurs is less obvious. It is possible that the domination of the two largest bodies in
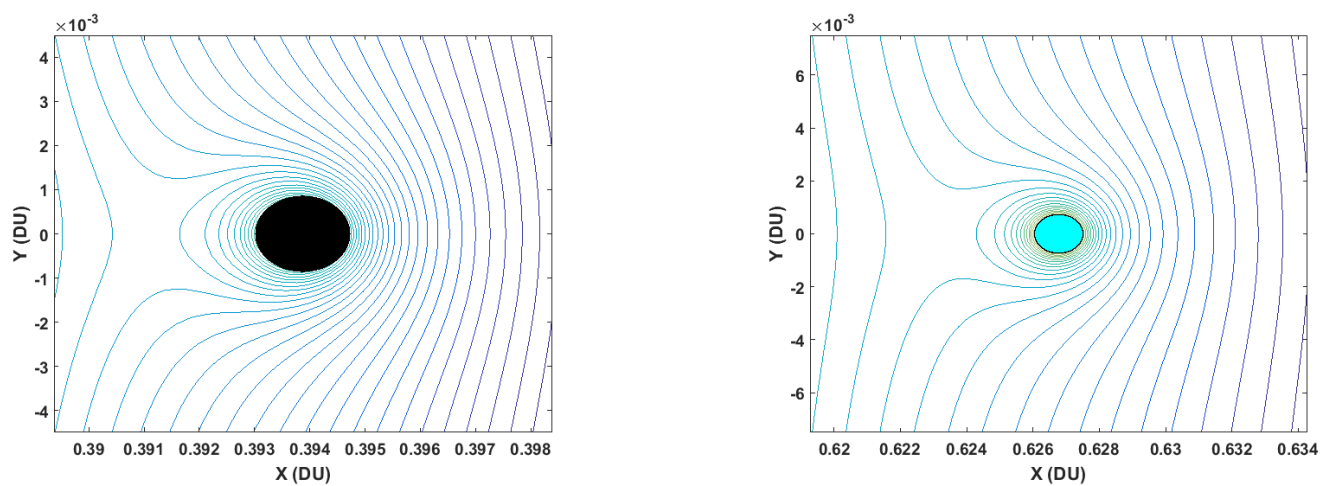

Figure 2.11: Jacobi Energy near Io and Europa

the system, coupled with the fact that Callisto, the only body relatively close in size to Ganymede, is much farther away, causes the energy of the system to behave fairly independently of the other moons. Perhaps Jupiter is so large, that only its largest will every have a significant impact on the surrounding energy space. More testing would be required to speculate further.

Regardless of why the system's intricacies behave as they do, it is clear that the derived equations for the Jacobi constant are feasible and their results behave similarly to the equations derived from the CR3BP. These equations open up a wealth of interesting a deep problems that should be explored further. Details and ideas on how this might be accomplished are discussed below in the section on future work. 
Chapter 3

\section{METHODOLOGY}

\subsection{Modeling Motion}

This section is a brief outline of the techniques and tools used to acquire the results presented in the following section. All code developed for this paper is available through Dr. Kira Abercromby, Associate Professor of Aerospace Engineering at California Polytechnic State University, San Luis Obispo.

For all dynamic simulations, the basic motion of a satellite is modeled in Matlab using ode45 with absolute and relative tolerances set to 1e-12. For normal orbital simulations, this level of accuracy is a little over-the-top, but for this system, motion is weakly chaotic, meaning that small differences in initial state can lead to big differences in the final orbit, making it important to tighten the propagation tolerances. Modeling the motion of the satellite as a set of first order equations is a trivial task.

To speed up the calculations and to simply any optimization problems, everything is translated into canonical units, where one distance unit (DU) was equivalent to the distance between the primary and the largest secondary, one mass unit (MU) was equivalent to the sum of the masses of the primary and the secondaries, and the period of the largest secondary body was set to $2 \pi$ TU. The end result of this is that Newton's Gravitational Constant becomes equal to 1, and the gravitational constants

of each major body $\left(\mu_{i / j}\right)$, therefore, is equal to each bodies canonical mass $\left(\mu=\frac{M_{j}}{M U}\right)$. See Table 4.1 for more details.

Another important step in simplifying the problem and speeding up calculations is utilizing the circular orbit assumption to track the secondaries. Instead of having to painstakingly propagate each body through, the position of each body could be 
treated as a simple percentage around a circle is has traveled in a given time. To figure out how fast each body moved, the mean motion is calculated based on the semimajor axis of the body. This mean motion of the largest secondary is then subtracted from each of these values to determine the relative mean motion of each of the bodies in the synodic frame, the frame of interest for these problems, and the frame in which the equations of motion of the small body were calculated. This leaves the primary and

the largest secondary fixed and causes the secondaries outside the largest secondary to move clockwise, those inside to move counterclockwise. All four of the Galilean moons move in retrograde orbits. It was assumed that every smaller secondary had an arbitrary starting position and rotated in the same direction in the inertial frame. This technique effectively turned the position of each of the secondary bodies into a lookup table or a couple lines of easy calculation, greatly speeding up the process compared to what would be required to propagate even a simple circular orbit with a standard orbital propagator.

\subsection{Calculating the Jacobian Energy and Characterizing Stability}

The Jacobi Constant was relatively easy to calculate once the equation for doing so are established. Since each energy map of the system corresponded to a unique configuration of the planet's moons, the calculations need to be done at every time step. To avoid the large computation time required, the areas of interest were limited to areas near the secondaries and to the entire area with the primary and secondaries included, the main difference between the two being precision and scale. Each of these were pushed forward in time with linear steps and analyzed independent of time.

To characterize stability in a system,analytically solving for points that were truly stable is most likely impossible because the nature of the problem is such that there is no fixed point that has zero acceleration relative to the system as there are in 
the CR3BP. Instead, there are only areas of very low relative acceleration. These areas were found and then observed over long periods to establish whether they were consistent, predictable, etc. A similar type of searching was used to determine which point, if any, had the lowest acceleration at any given time. This points were tracked over time as well, giving insightful looks into how the perturbations from the other large bodies pulls around the would-be Lagrange points.

\subsection{Manifold Investigation}

Halo orbits, trajectories that periodically orbit Lagrange points, in 3 body systems require a very high level of accuracy in order to maintain. Since there are perturbing bodies in motion in $\mathrm{N}$ body systems, one could imagine a time varying solution to repeating halo orbits, adding a periodic perturbation with a frequency equal to that of the associated body, or a solution in which some minimum $\triangle v$ could be allocated to correct the orbit each revolution, but both of these are well beyond the scope of this thesis. In order to model halo orbits with $\mathrm{N}$ bodies, the extra bodies are simply ignored to get an initial guess and then put back into the dynamics to converge upon a solution for halos in a given orientation of the system. The particular halo orbits analyzed are found using the third order approximation technique outlined by Richardson[8] and converged upon using a simplistic shooting method that, although more difficult to control and slower than the state transition matrix modification used by Howell [5], can use CRNBP dynamics. Since propagating manifolds required knowledge of the Jacobian of the system at the Lagrange point being orbited, the associated state transition matrix was also calculated in the three body system. It would be possible to take the point of minimum acceleration discussed above and find the Jacobian there, but for simplicity and speed, this technique was less desirable. It is important to note that these manifolds are therefore not invariant manifolds 
since they are constructed with 3 body assumptions and propagated with $\mathrm{N}$ body assumptions. Instead, these manifolds represent a perturbed form of the invariant manifolds from the three body system.

Low energy transfers were constructed similarly to halos and manifolds. When manifolds that began under the influence of the Jovian $\mathrm{N}$ body system exited the sphere of influence of Jupiter, the trajectories were switched to CR3BP motion in the Sun-Jupiter system and visa-versa. 


\section{Chapter 4}

\section{RESULTS}

Before going into the detailed results, it is also important to lay out the basic foundation on which each of the sets of tests was run. Since the underlying assumption that the largest mass is approximately at the barycenter of the given system, and all of the secondaries are in circular orbits around that barycenter, it was important to find systems where this was not far from the truth. The first major contenders were the gas giants, since they are enormous and tend to have lots of large bodies orbiting them, to act as case studies. Saturn was eliminated because of the difficulty modeling the effects of its rings, though this could be added on later as a perturbation to the given system. Saturn's mass ratios are very interesting, being much closer than those in other systems - on the order of the ratio between the Earth and its moon's mass - making this an unfortunate exclusion. The remaining gas giants Jupiter, Neptune, and Uranus - were all viable. Jupiter has 4 of the largest 20 objects in the solar system within it's influence, Neptune two, Uranus four, but the system is more applicable to Jupiter because of practicality. Missions to Jupiter are simply much more feasible with current propulsion technology than missions to Neptune or Uranus, with the latter potentially requiring decades of flight time. Serious consideration was also given to analyzing the solar system. Not only would this be one of the most interesting systems, it is an excellent example of a system that meets the restricting assumptions, but, like Uranus and Neptune, it was not chosen because any transfers that could potentially utilize, or visit other planets, would be inherently lengthy and therefore less useful. Jupiter and its four largest moons (the Galilean moons: Ganymede, Europa, Io, and Callisto by mass) made up the system considered in the following analysis (unless otherwise stated). Some numbers were run from 
the CRNBP against the Earth-Moon CR3BP as sanity checks, but the distance of the Earth-Moon barycenter from the Earth makes the assumptions of the former less likely to be accurate than those of the latter. The majority of experiments were limited to the Jovian system and the Sun-Jupiter system with the Sun-Jupiter system modeled as the sum of major five masses in the system centered at Jupiter's position.

The first ubiquitous step in analyzing theses systems was to put all the associated constant values into canonical units. Though it was more difficult to manipulate these to measure distances in terms of mass ratios (as done in the CR3BP), putting all units into canonical form simplified the calculations and sped up computation. For this thesis, a distance unit (DU) was defined as the distance between the largest secondary and the primary, a time unit (TU) was defined such that the period of the largest secondary was 1 , and a mass unit (MU) was defined as the sum of the masses of the primary and the secondaries. Each unit conversion is given in Table 4.1. This

Table 4.1: Canonical Conversions

\begin{tabular}{|c|c|c|c|c|}
\hline \multicolumn{2}{|c|}{ Jupiter-Galilean System } & \multicolumn{2}{|c|}{ Sun-Jupiter System } & \multirow[b]{2}{*}{ Derivation } \\
\hline Unit & Value & Unit & Value & \\
\hline Mass (1 MU) & $1.8984 \mathrm{e} 27 \mathrm{~kg}$ & Mass (1 MU) & $1.9907 \mathrm{e} 30 \mathrm{~kg}$ & $\sum m$ \\
\hline Distance (1 DU) & $1.0704 \mathrm{e} 06 \mathrm{~km}$ & Distance (1 DU) & $7.7830 \mathrm{e} 08 \mathrm{~km}$ & $\left\|r_{2}\right\|$ \\
\hline Time (1 TU) & $9.8382 \mathrm{e} 04 \mathrm{~s}$ & Time (1 TU) & $3.4463 \mathrm{e} 07 \mathrm{~s}$ & $\frac{P_{2}}{2 \pi}$ \\
\hline
\end{tabular}

system is very natural since it has the 1 st moon fixed on the coordinate $(1,0)$ moving at $\frac{1 D U}{1 T U}$ with a rotational rate of $2 \pi \frac{r a d}{T U}$, meaning it completes its rotation in $1 \mathrm{TU}$. From there, everything can be understood on a simple relative scale. 


\subsection{Stable Regions, and Quasi-stable orbits}

The goal of this section is to show how the $\mathrm{N}$ body system equations of motion can be applied in ways similar to those of the 3 body system to yield locations that are more or less stable - locations like Lagrange points. This is section also looks at the possibility of created and mapping unique periodic and quasi-periodic orbits within the $\mathrm{N}$ body system. Although halo orbits probably fall more accurately under the title of "quasi-stable orbits," since they are the basis for manifolds and low energy transfers, they are discussed at length in their own section.

In order to find Lagrange points, or stable points like them, the first step is to see if it is possible to find an analytic solution to the equations of motion with the velocities and accelerations set to zero. This proves to be incredibly difficult, if not impossible, due to the extra term added by perturbation from the extra bodies. Not only that, but any solution found will only be a valid solution for one given planetary configuration, and will therefore have to be reevaluated constantly as the planets process. For this reason, a numeric search seems to be the more reasonable route. Not only will it avoid the mess of trying to solve what is at least a very difficult set of equations to solve, but, with modern computing, a numeric search is quick and effortless even for a large number of time steps so long as there we have a ballpark guess of where the points of minimum acceleration in the system are located. Using the Lagrange points of the associated 3 body system as a starting guess works well.

By doing a refined grid search and narrowing down a simple gradient plot of the acceleration in the n-body system relative to the zero-velocity space, the point of minimum acceleration (it is likely that there is no point of zero acceleration) can be found continuously as the bodies in the system are propagated forward in time. The results are shown for the Jupiter-Ganymede pseudo- $L_{1}$ point in Fig. 4.1 and Fig. 4.2 for a high precision, short duration propagation (high precision in terms of the detail 
of the grid search, not in terms of the method of propagation) and low precision, long duration propagation respectively. Both of these plots show that the movement of the point is consistent, but is constrained to a certain degree. It's hard to say whether the area that is cut out by the movement of the point represents an area that it would be possible to stay within for a very long period without orbital corrections or whether it is simply impossible to stay anywhere in the system without constant corrections. The latter seems more likely, though further investigation would be required to reach a definitive answer. Regardless, the motion of the point of minimum acceleration between the two largest bodies, the most impacting gravitational bodies in the system, moves on the order of hundreds of kilometers in several months simply due to the perturbations from the other secondary bodies. The motion of this point also seems bounded and periodic. This opens the door to a entirely new kind of halo orbit that would never occur in the CR3BP system. Even with incredible precision, traditional halo orbits in any $\mathrm{N}$ body system would decay very quickly and would require higher amounts of $\Delta v$ to maintain than their theoretical counterparts from the CR3BP. There might be a solution to linearized motion for halo orbits about an oscillating point like the one being shown, but that would be an entire project in itself. It also seems that more simple periodic orbits, like the ones shown later in this section, would also naturally diverge, meaning truly periodic orbits in any $\mathrm{N}$ body system would be impossible. Even in the circular restricted three body case, it is very difficult to find orbit that are periodic for extended periods without going to extremely high accuracies. Halo orbits, for example, will often diverge within relatively short time spans if their trajectories are not accurate on the order of meters. The N-body system exhibits this kind of behavior as well, but to a larger extent - it is easy enough to find orbits that have periodic or quasi-periodic properties, but it is very difficult to find trajectories that repeat on large time scales. It is likely that the $\mathrm{N}$ body system's perturbations keep truly periodic orbit from occurring due to the fact that 


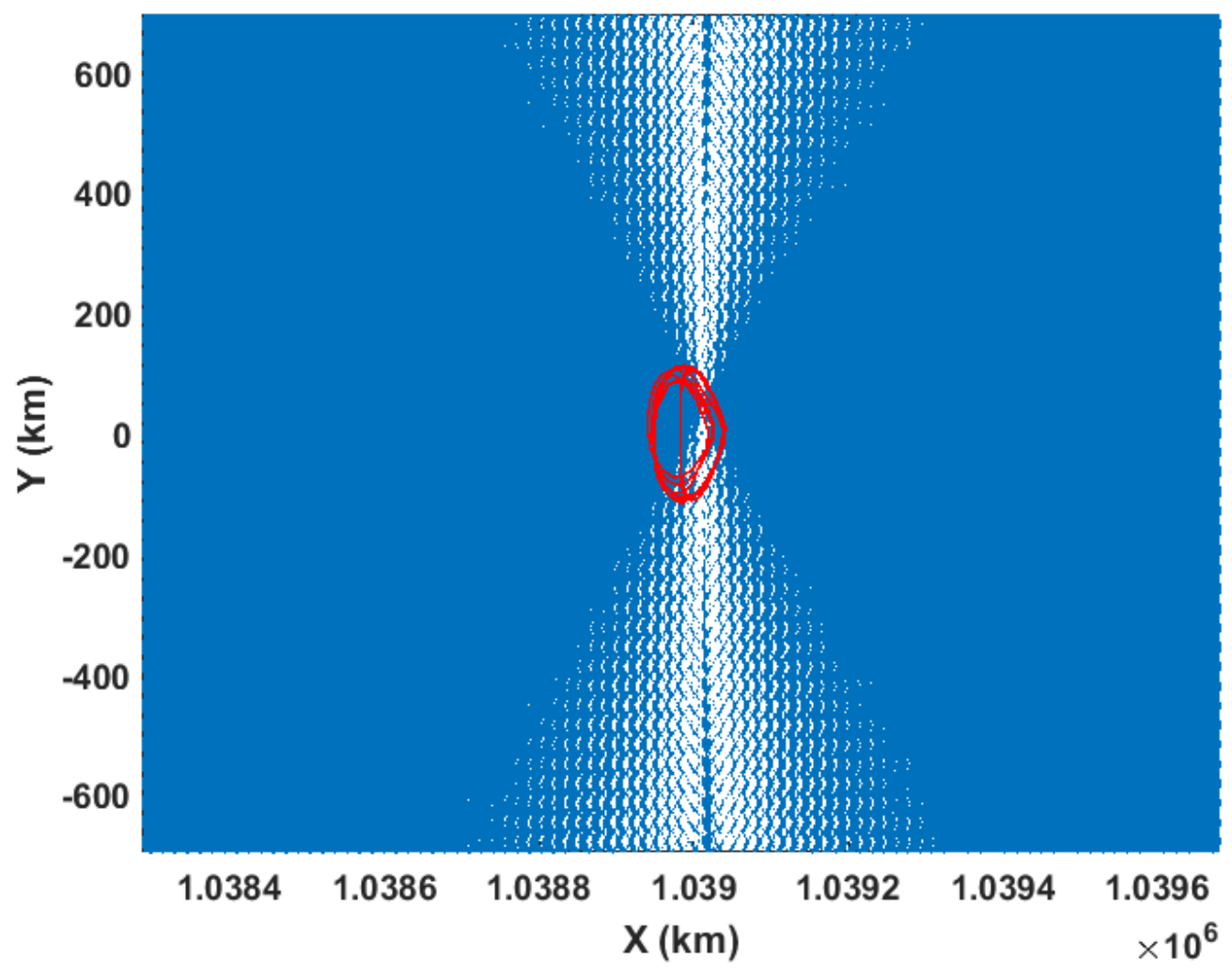

Figure 4.1: Motion of the "Pseudo-Lagrange" Jupiter-Ganymede L1 point for 1 Week

it would take astronomical time periods for the secondaries of the system to line up in any given geometric orientation multiple times, but finding orbits that are close to periodic turns out to be trivial. To find these quasi-periodic orbits, an arbitrary location is picked and a simple shooting method converges on any orbit starting in the given position that would return to the initial position and velocity. To help the solver, only positions along the $\mathrm{x}$-axis are chosen and initial guesses for the starting velocity are only in the y-direction. This, coupled with a cost function that valued orbits that crossed the $\mathrm{x}$-axis with velocity vectors consisting of only y components, is able to converge on dozens of different and telling orbits. The first example given in Fig. 4.3 shows one such orbit.

The orbit is unique in two important respects. First, it is not symmetric about 


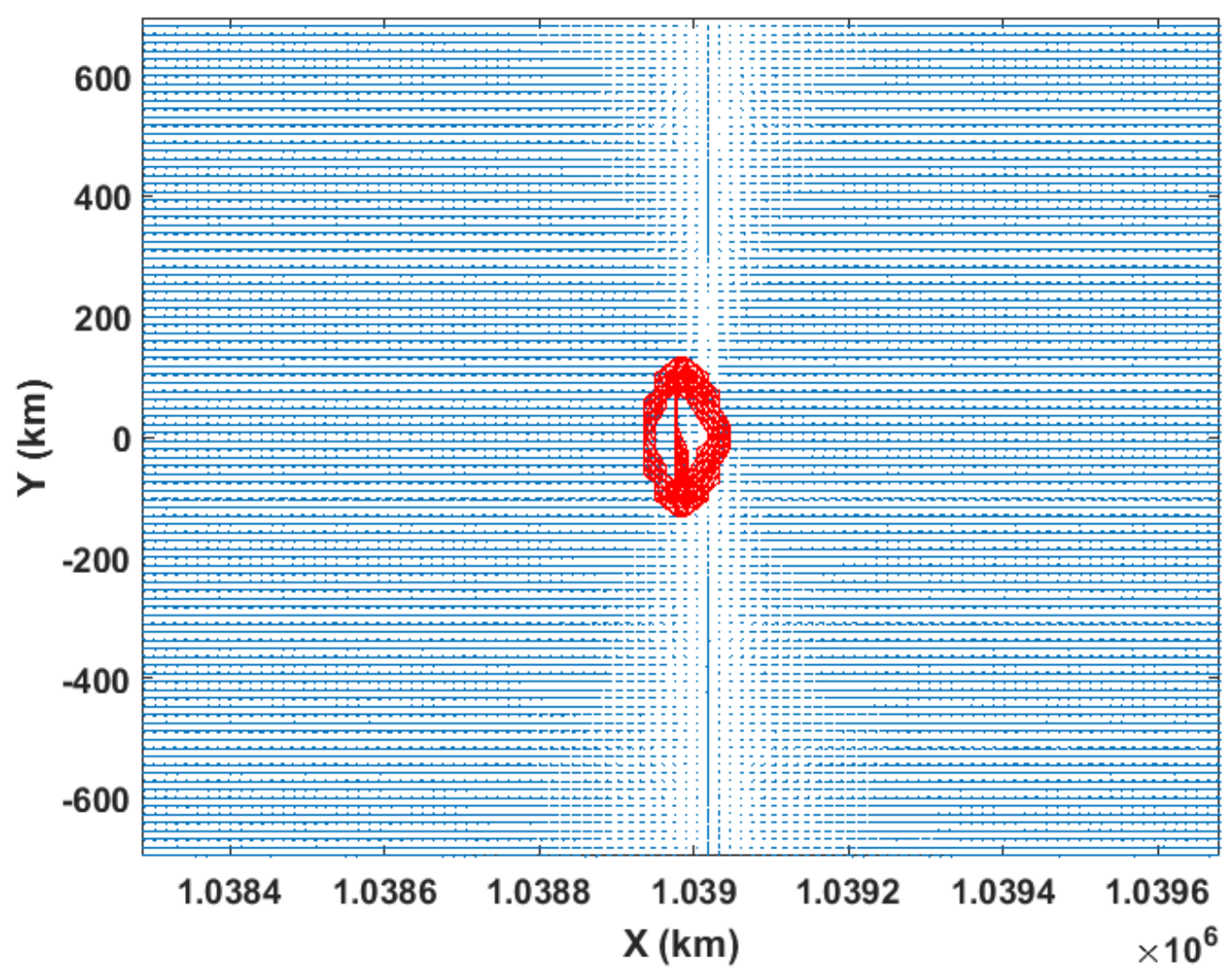

Figure 4.2: Motion of the "Pseudo-Lagrange" Jupiter-Ganymede L1 point for an extended period

the x-axis, meaning that it is boosted by one of the secondaries (Ganymede). Second, it has a decent range of possible observation locations relative to the Jovian system. This orbit passes close enough to get close up images of every single major moon, a very close look at Ganymede, as well as a variety of different looks at Jupiter itself. The fact that this orbit uses a flyby of Ganymede also means that this orbit would consistently allow for a close look at the moon without having to use $\triangle v$ to slow down a spacecraft into an orbit around the body and it would allow for the ability to change trajectories to more closely examine another moon or the planet itself without using the fuel required to exit a low orbit around the moon. The second example (Fig. 4.3) shows that it is possible to construct similar orbits in three dimensions, though did prove to be more difficult. Trajectories that satisfied the requirements in 
three dimensions could not have the additional requirement that they would intersect the $\mathrm{x}$-axis with no $\mathrm{x}$ and no $\mathrm{z}$ velocity, making it more difficult for the function to converge on possible solutions. More complex and interesting three-dimensional periodic orbits are given below in Fig. 4.4. The usefulness of these orbits beyond their
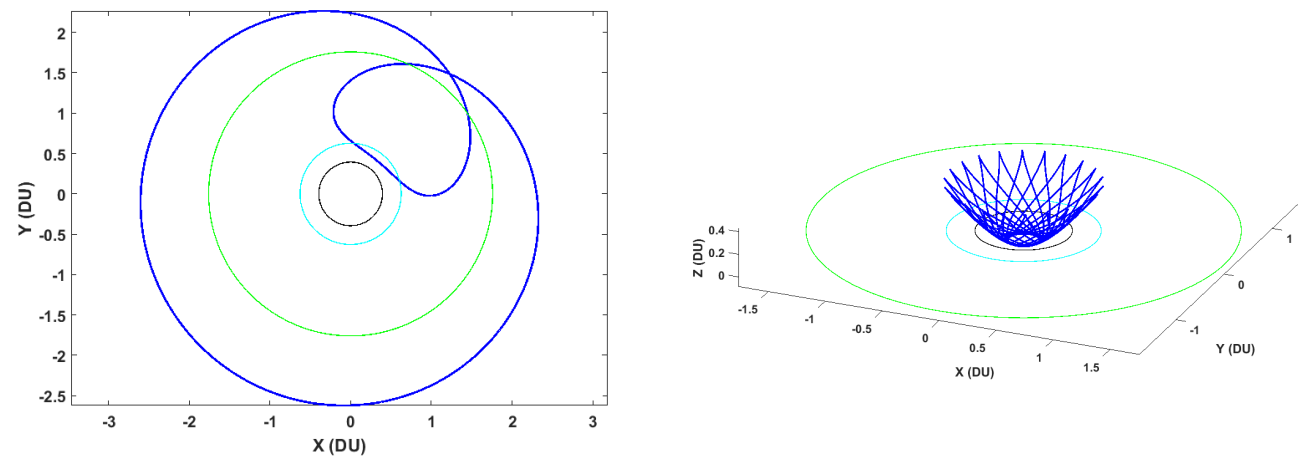

Figure 4.3: Examples of quasi-periodic orbits

aesthetic value is probably questionable, but they might be able to provide interesting viewing angles of the planet and its moons that planar orbits would be unable to do. It is also possible that these orbits could provide interesting measurements of Jupiters enormous magnetosphere. Regardless of their legitimate practical implications, both of these orbits demonstrate that nearly periodic orbits are possible in three dimensions in the CRNBP. Example 3, given in Fig. 4.5, is an orbit more reminiscent of those
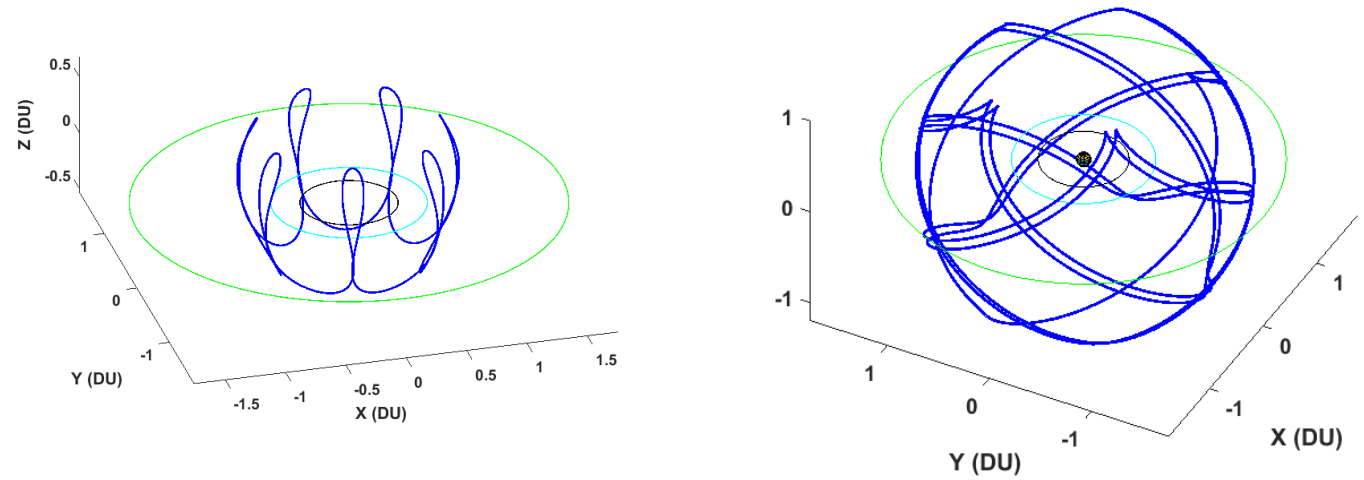

Figure 4.4: Out-of-plane quasi-periodic orbits 
that are commonly found in the CR3BP. It is symmetric about the $\mathrm{x}$-axis, it starts and ends near one of the Jupiter-Ganymede Lagrange points, and it goes around the moon once per period before its exodus to the L3 point and back. All of these orbits, however, are only nearly periodic, not truly so. Every one degrades or is shifted asynchronously with its period over time, they are simply shown for a single orbit to emphasize how close they are to being truly periodic. The fourth example in the same figure demonstrates this property. The most telling aspect of this orbit is the nonuniform separation of the segments of each consecutive pass. If the orbit was periodic, even over some long time span, there would a consistent change in the flight path on each pass which is clearly not the case. Whether or not this makes the orbit more or less useful would be dependent on the mission, but from this two generalizations can be gleamed: one, perfectly repeating orbits in the N-body system are either nonexistent, very difficult to find, or only happen over extremely long time periods, and two, the general behavior of a trajectory is only affected by the perturbational pull of the secondaries when the orbit is sensitive to small changes. Fig. 4.6 shows one such case of the latter behavior. This figure shows an orbit around Ganymede that diverges
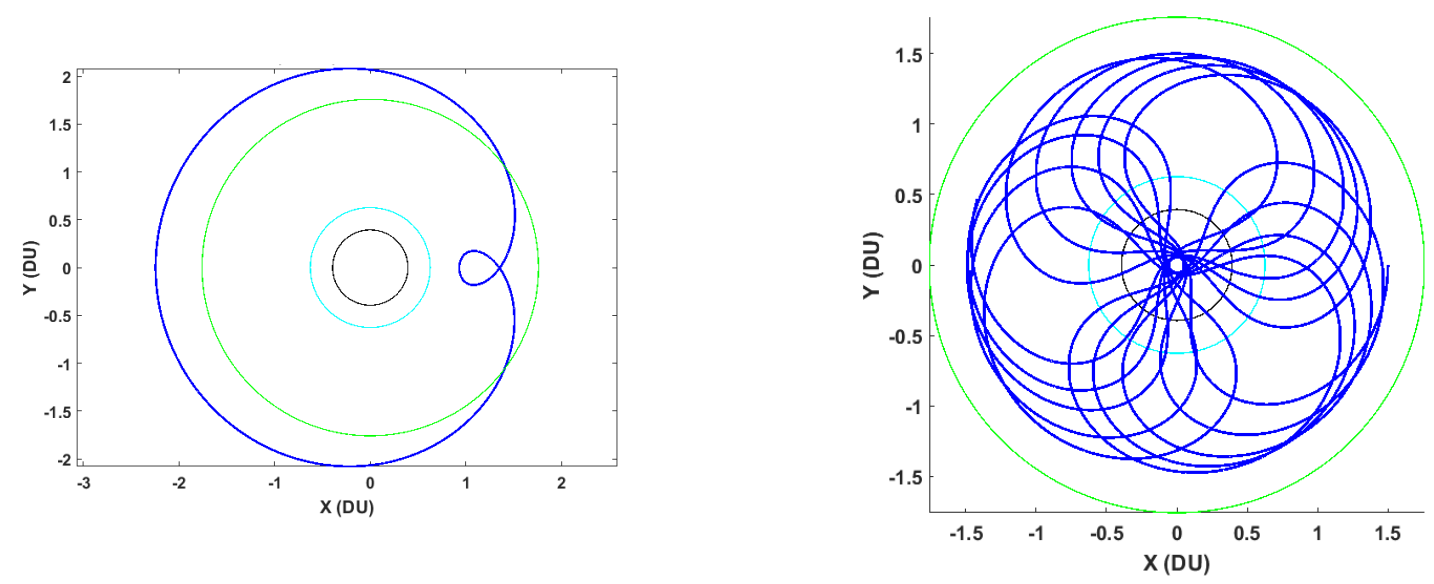

Figure 4.5: Planar periodic orbits

after a single pass despite the fact that it meets the convergence criteria for periodic 
orbits specified by the cost function used for all of the other given examples. Although it isn't shown, the divergence causes the orbit to change dramatically depending on the precision of the propagation. In one case, it will quickly crash into Ganymede, and in another, it will escape its immediate influence. In both cases, the first pass appears as though the trajectory will be stable for at least some amount of time. The sensitivity of certain orbits should not be overlooked for this reason - although there might exist families of orbits that are seemingly stable in the 3 body system, the pull from other secondaries can easily perturb a body enough to drastically alter its final state. Take the trajectory shown in 4.7 as a final proof of the sensitivity of orbits in

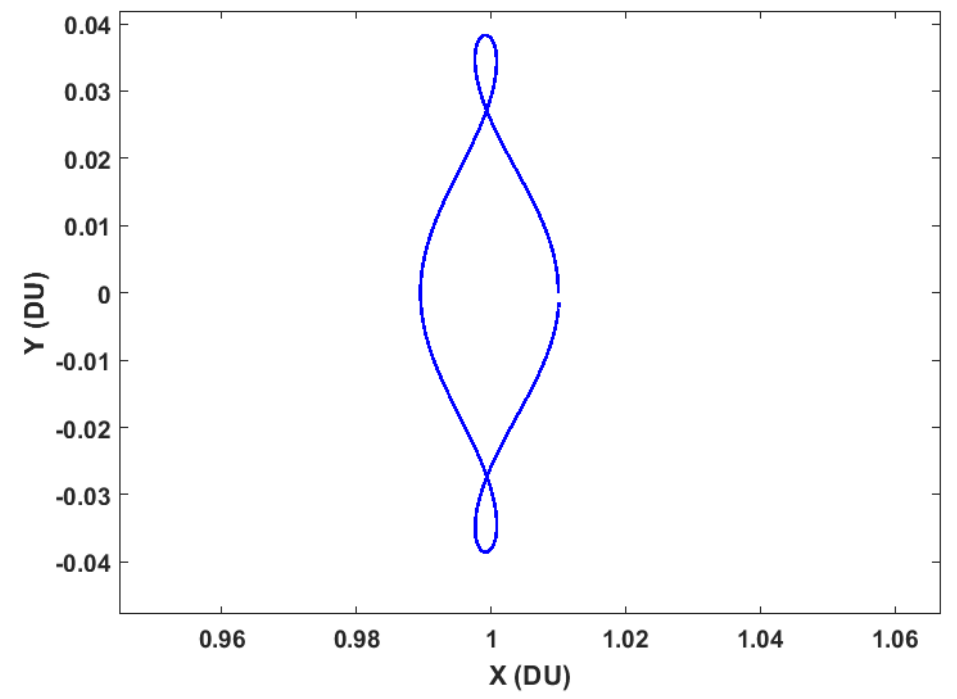

Figure 4.6: Trans-Ganymede Periodic Orbit

the CRNBP system. This orbit is a surprising result because it satisfies the simple 2 dimensional requirements of periodicity, namely that the velocity was purely in the $\mathrm{y}$-direction when the body crossed the $\mathrm{x}$-axis, despite being a seemingly chaotic 3 dimensional orbit. Starting around .8 DU along the x-axis with a small z component and an initial velocity only in the y-direction, the body orbits in a strange, nearlycircular orbit for two or three passes and then it started to slowly diverge. After a 
small divergence from the apparently circular path, the divergence became dramatic, causing the body to show changes in its z component much larger than it's initial position and velocity would suggest. It's important to remember that this trajectory
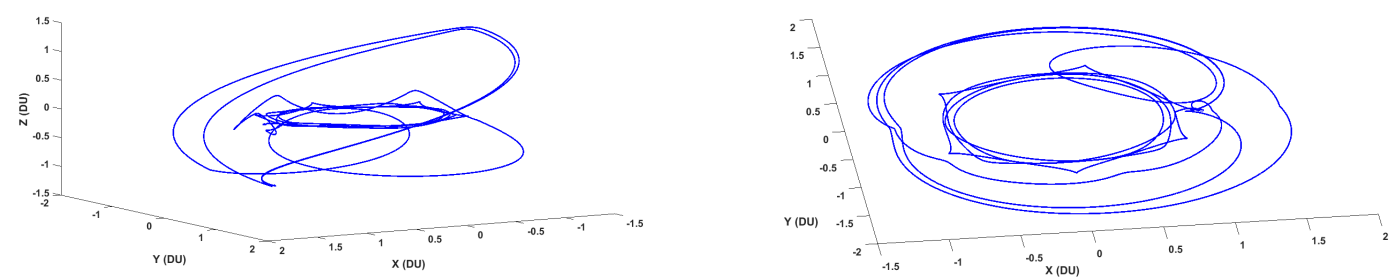

Figure 4.7: A highly irregular orbit with unique viewing coverage properties

is accomplished with no thrust or $\Delta v$ whatsoever, it occurs naturally in the system. Changes of this magnitude are generally only possible in the CR3BP with flybys or thrust, both of which are more expensive from a mission standpoint than passively allowing perturbations to take the spacecraft where they will. This kind of orbital transfer includes a increase in the semimajor axis, a change in eccentricity, and an otherwise infeasible jump in inclination, all of which take a significant amount of fuel in a two body case, accomplished in the Jovian N-body case simply through the perturbational pull from the smaller secondaries and unique initial conditions. It doesn't take a lot of imagination to see the various ways in which this kind of orbital transfer capability would be invaluable for any mission design.

\subsection{Halo Orbits and Manifolds}

The goal of this section is to look at and analyze the various methods for calculating and propagating halo orbits in the CRNBP. It also introduces one possible way of maintaining those halos. There are a number of methods for approximating halo orbits in a three body system. One of the more famous methods was developed by Richardson?? in 1978. His third order approximation to halo orbits around the 
collinear Lagrange points in a 3 body system is simple enough to implement and can be used as a basis for higher order approximations, such as those outlined by Howell??. Both of these techniques are successfully implemented in the N-body system, but each solution is only valid for one geometric configuration of the secondaries and will quickly diverge when propagated with CRNBP dynamics. To solve this, instead of using Howell's method of converging on halo orbits from Richardson's approximations in 3 body systems, a simple shooting method optimization is employed with Matlab's fmincon function. The method involves picking a starting point on the $\mathrm{x}$-axis depending on whether the halo was in a northern or southern family, and propagating a body forward to the next x-axis crossing. Since halo orbits cross the $\mathrm{x}$-axis with only velocity in the $\mathrm{y}$-direction by definition, the cost function of the optimization is a sum of the $\mathrm{x}$ and $\mathrm{z}$ velocities at the axis crossing. For most cases, the initial $\mathrm{x}$ position from the third order approximation is fixed and the initial z position and y velocity are subject to change. This method turns out to be very effective though it doesnt entirely solve the problem. Despite the fact that these halos are being approximated with CRNBP dynamics, the constantly changing positions of the secondaries make it impossible for these approximations to find halo orbits that are stable for more than a few revolutions.

The question then becomes: Is halo orbiting possible at all in systems where there are more than 3 bodies? An entire thesis could be written on the possible ways of optimally maintaining halo orbiting in this system. For simplicity, a method of continuously correcting the halo orbit is employed whereby a small impulse burn is performed each half orbit so that the next half orbit has the properties of halo orbits used in the optimization scheme described in the previous paragraph. While it doesn't consistently maintain the original halo orbit, this technique does use less $\Delta v$ than attempting to force the same halo over and over. Largely, this method proves successful, if not pragmatic on a mission level, and it opens the door to a potential 
study on the possibility of more and less stable regions in families of halo orbits.

The first example of this method of corrected halo-orbiting is both generic compared to the other solutions, and, because of this, informative. The amount of $\Delta v$ required to maintain halo orbiting is on the order of $5 \frac{\mathrm{m}}{\mathrm{s}}$ per correction initially, averaging out to approximately $2 \frac{\mathrm{m}}{\mathrm{s}}$ after 20 or so orbits. The results for the required change in velocity are summarized in Fig. 4.9. It is also interesting to note that the change in each successive halo is less and less as the number of orbits progresses. Figure 4.8 shows a tendency of the halo to correct to a specific region instead of staying near its original position. Initially, this phenomenon was much more exag-

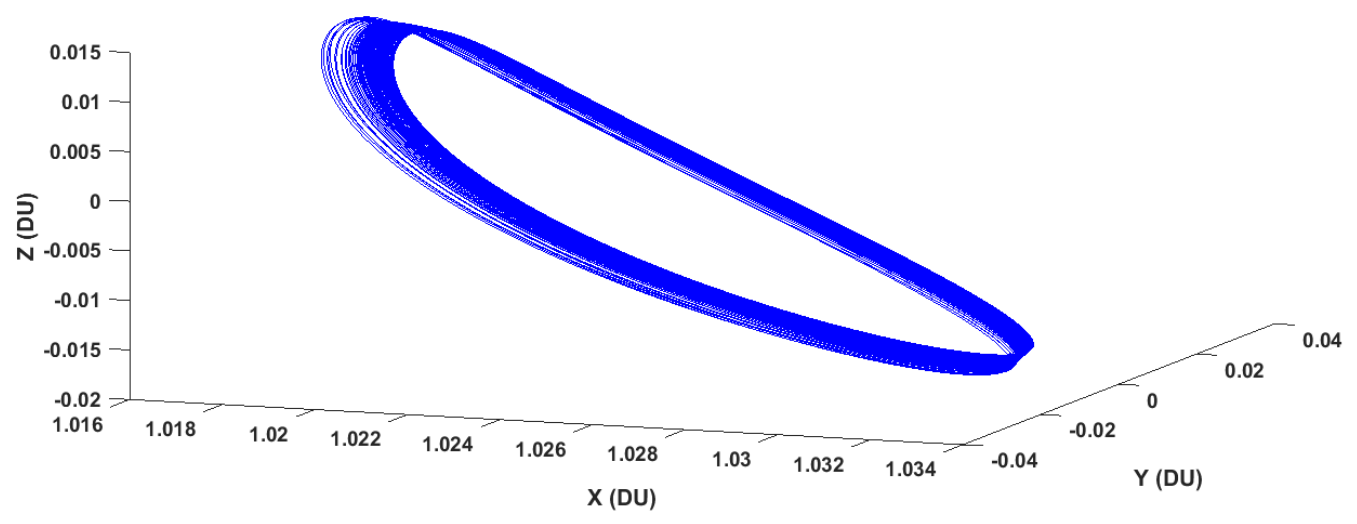

Figure 4.8: Corrected quasi-halo orbiting

gerated, with the halo bouncing up and down between corrections, so each guess for the direction of each correction was modified so that it was in the opposite direction as the previous correction. The thought was that this would keep the orbits from rapidly diverging by forcing the optimization scheme into solving for solutions that counteracted one another instead of building on each other. This technique works for most scenarios, and is used for all of the figures shown. The appearance of convergent tendencies from this technique was unexpected. 


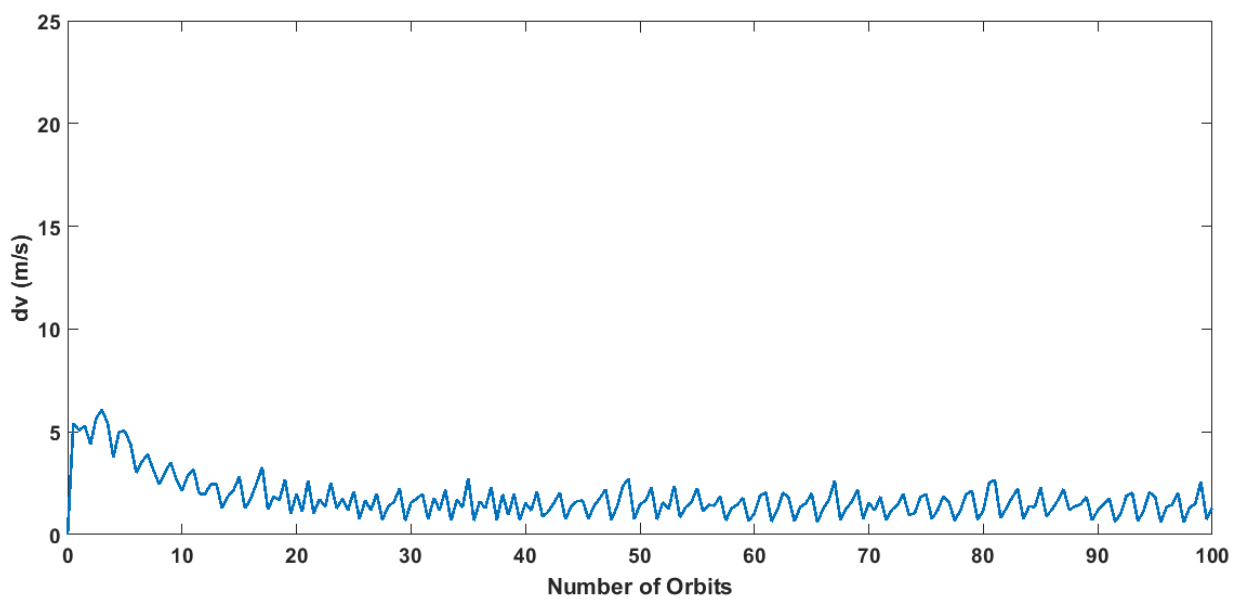

Figure 4.9: $\triangle v$ required to correct the Halo

As Figures 4.10 and 4.11 show, trying to force each half orbit to correct in the opposite direction of the previous correction can manifest with the appearance of stable set of halos while having erratic changes in the direction of the corrections. The graph on the right in Fig. 4.11 gives the $\Delta v$ for each correction by the direction of the burn. The burns seem exaggerated and wobble quite a lot, despite the fact

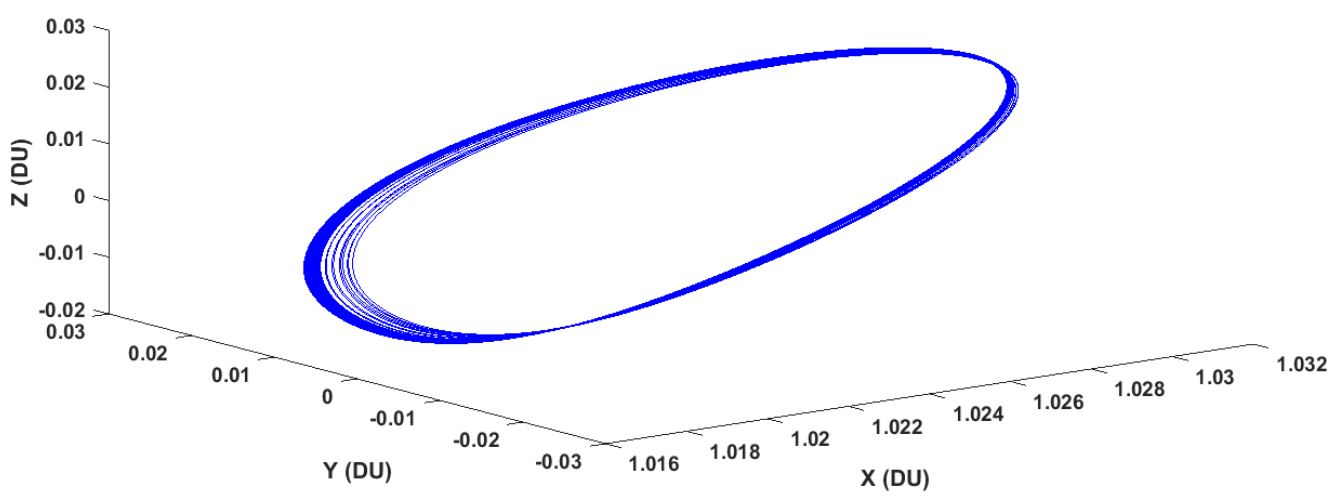

Figure 4.10: Corrected quasi-halo orbiting with wobbling

that the orbit appears to stabilize with an average correction $\Delta v$ of around 2 or $3 \frac{m}{s}$. 

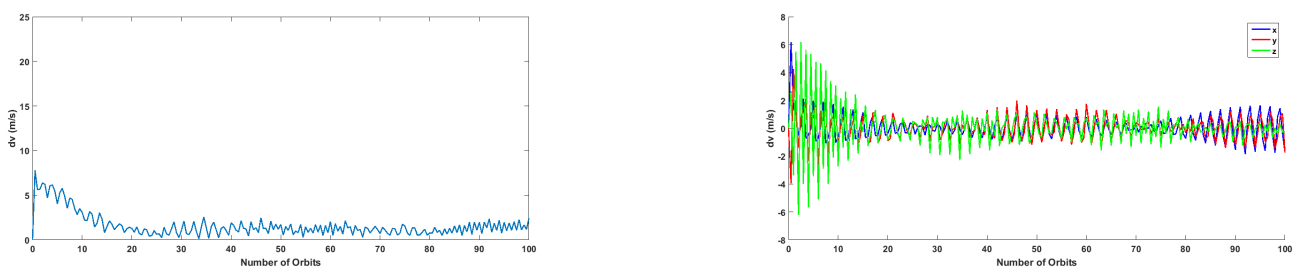

Figure 4.11: $\triangle v$ for the wobbling halo

It's unclear whether this is indicative of a poorly approximated halo, or a poorly designed method of maintaining a halo in the system. It is possible that there are a large number of different solutions for each half-orbit approximation and the method by which one solution is favored over another causes the lifetime of the burns to be heavily dependent on the direction of first burn. The burns represented in figure 4.11, for example, start off with a relatively large burn in the z-direction and maintain burns with a dominant z-direction throughout the 100 orbit cycle, while those shown in figure 4.13 begin with large, erratic burns mostly in the x-direction, somewhat in the z-direction, and not at all in the y-direction, and continue this behavior for the entire simulation. This behavior, however, is not consistent throughout test cases, and does not allow for definitive conclusions on whether or not the trends of these halos is due to the methodology by which the direction of the corrections is found or if it is simply due to the dynamics of the system. Another interesting trend of the halo in figure 4.12, besides its consistently erratic corrections, is the instability of the halo itself. Although other test cases seem to want to come to a certain halo or some small set of halos that are similar to one another, this halo seems to move through a larger range, oscillating back and forth for the time of the simulation. It is likely that this orbit will eventually completely diverge from its initial halo family, but more tests would be required to confirm this.

A great example of a halo that eventually diverges is given in the next figure, Figure 4.14. Despite the method for correcting each halo orbit, this halo was even- 


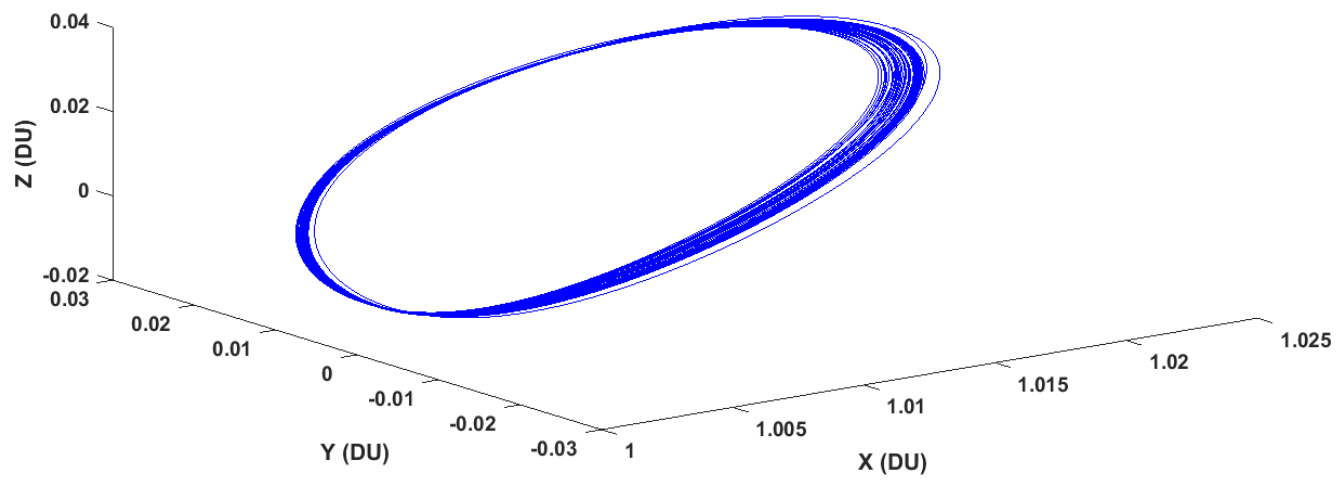

Figure 4.12: Unstable corrected quasi-halo orbiting
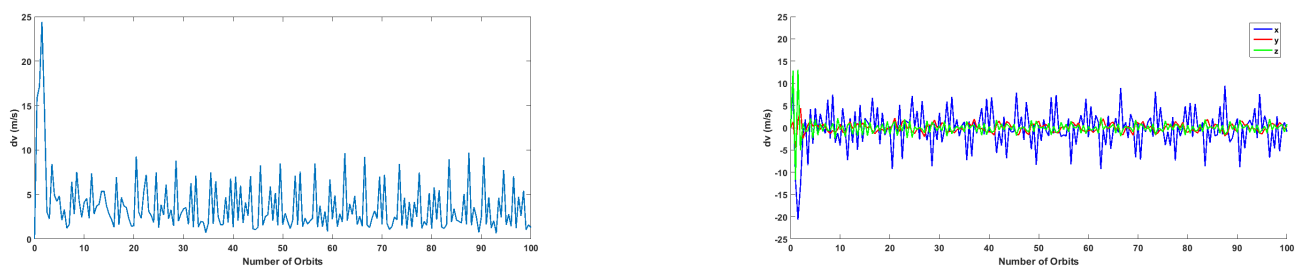

Figure 4.13: $\triangle v$ for the unstable halo

tually flung out of the region where halo orbiting would be possible at all. Due to the repeated success of avoiding this scenario, it is safe to say that this divergence is most likely due to the inherently unstable nature of the CRNBP system. For whatever reason, in this example, the perturbations from the extra bodies in the system worked to pull the body away from its family. It is possible that there are points in the halo orbit that are more unstable than others, points where small perturbations can cause a satellite to diverge more easily than doing so at other points in the orbit, and this orbit just happens to reach one of those points at an inconvenient time. It is also possible that this is a coincidence of the geometry of the planets, but this is unlikely since all of the examples given in this section have the same starting planet geometry throughout their propagation. The divergence of this orbit is also very 
apparent in the plot of the $\triangle v$, where the required change in velocity jumps up an order of magnitude near the end of the simulation.

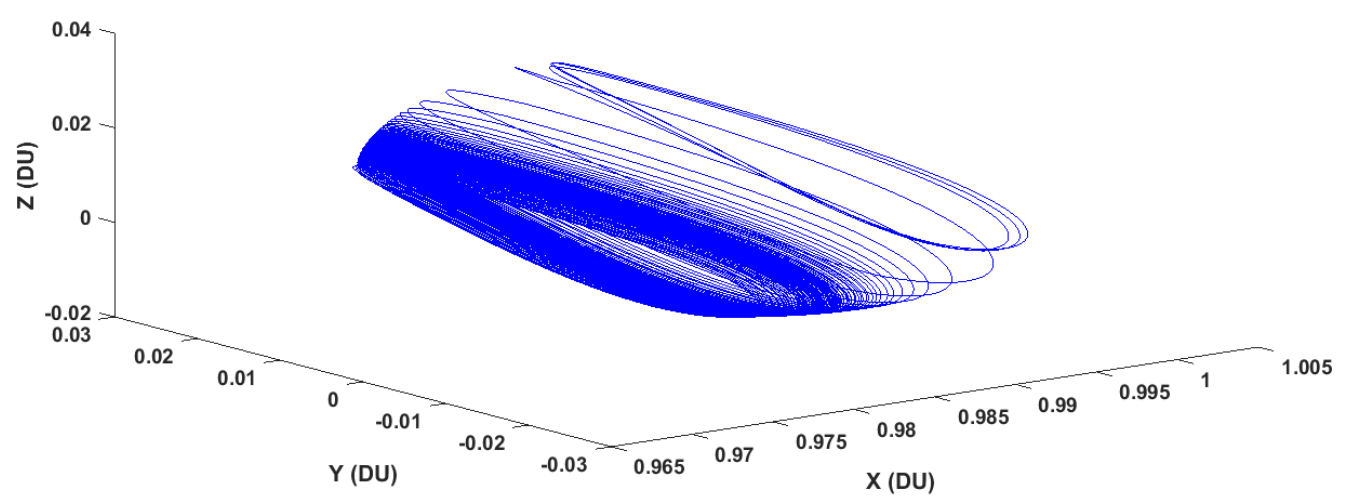

Figure 4.14: Divergent corrected quasi-halo orbiting

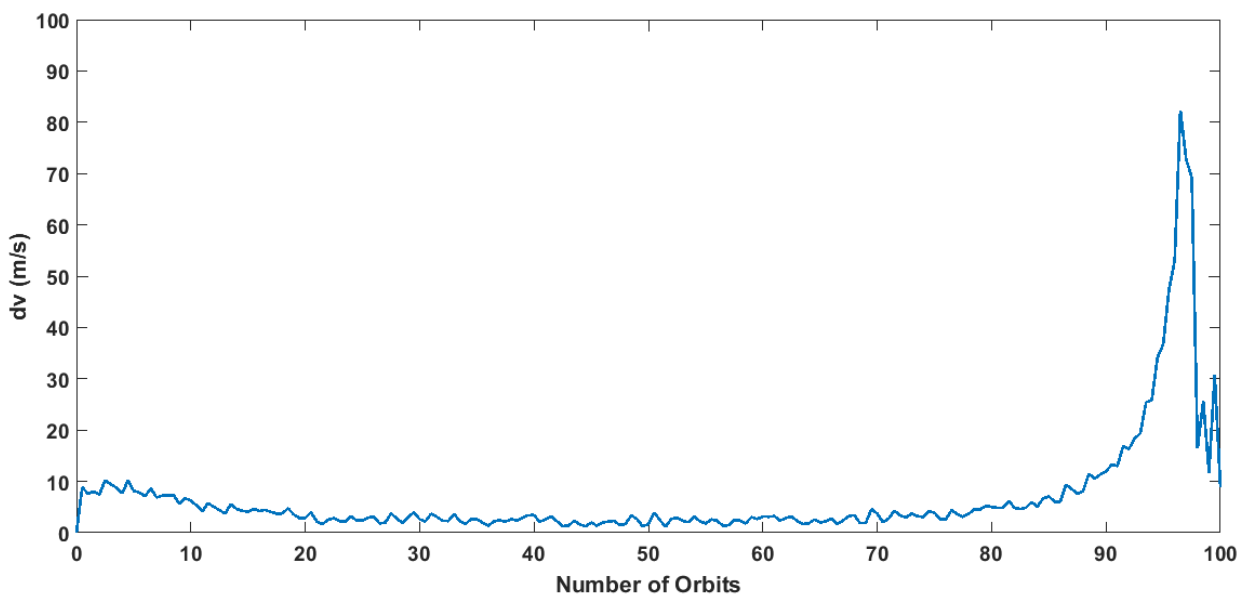

Figure 4.15: $\triangle v$ for the divergent halo

The divergence of this orbit seems more coincidental than anything. Some orbits, such as figure 4.16, stay in almost the exact same place that they started and require 
almost no correction to maintain, and some, such as figure 4.18, converge with a similar dramatic flare as the divergence of the example in figure 4.14.

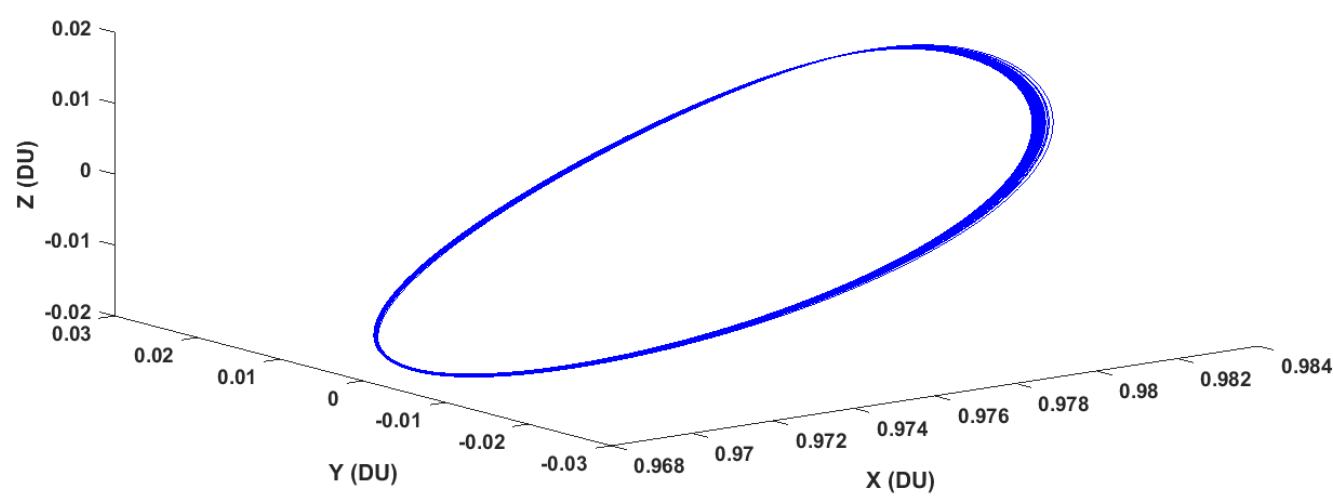

Figure 4.16: Highly stable corrected quasi-halo orbiting

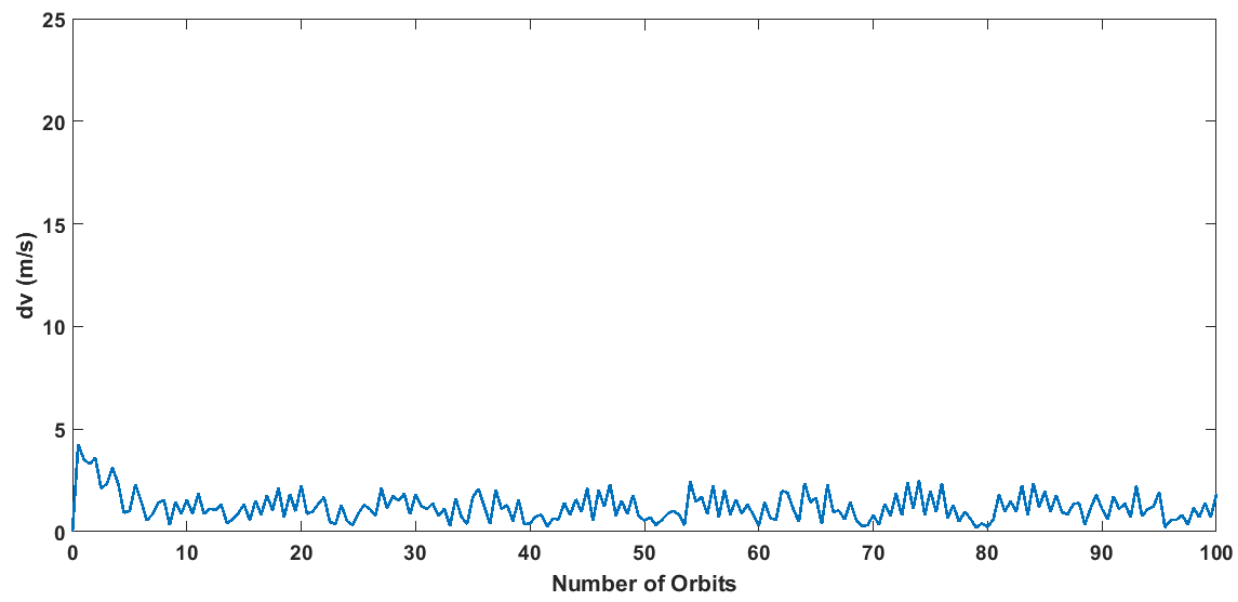

Figure 4.17: $\triangle v$ for the stable halo

The more profound conclusions of this quick look at halo orbits in the CRNBP are still unclear. The apparently sporadic divergence or convergence of certain halos 


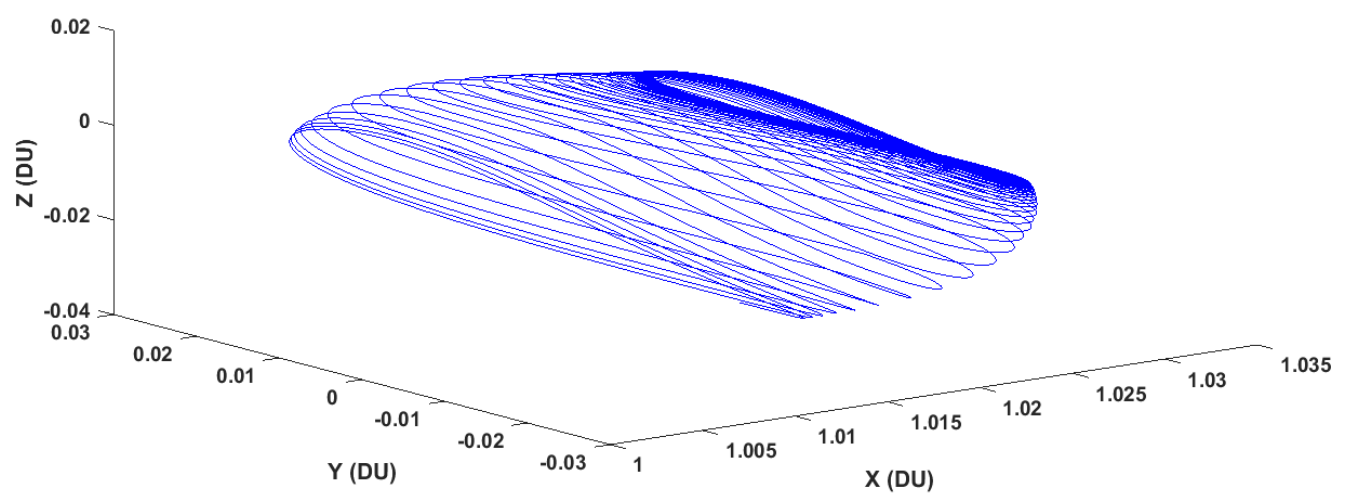

Figure 4.18: Convergent corrected quasi-halo orbiting
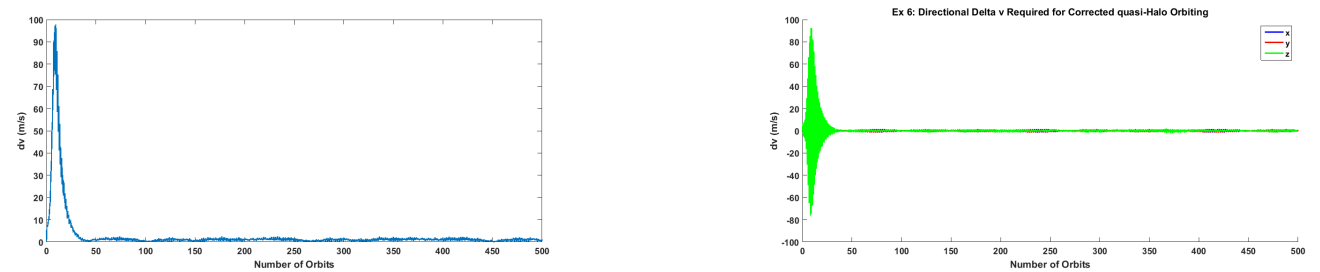

Figure 4.19: $\triangle v$ for the convergent halo

could be due to the method by which the halos are maintained or it could be a side effect of the $\mathrm{N}$ body perturbations. If the latter is the case, there is a possibility that there exists smaller sub-families of halo orbits in $\mathrm{N}$ body systems that are more or less stable over time. Either way, there is undoubtedly a better way to correct these halos, whether that is with less frequent burns, with more intelligent burns, or with continuous burns, as with electric propulsion. The potential for any or all of these is exciting.

Halo orbits, however, are just the first step in planning complex transfers in the CRNBP. Once some kind of halo orbit is determined, the next step is to produce manifolds from that halo, from manifolds, low energy trajectories, and from low energy trajectories, highways. The process for producing manifolds in the CR3BP is well understood and easy enough to implement. Even Io, which is very close relative 
to Jupiter's other moons and is much smaller than Jupiter in terms of mass, is capable of producing stable halo orbits, and stable and unstable manifolds when analyzed with the CR3BP.
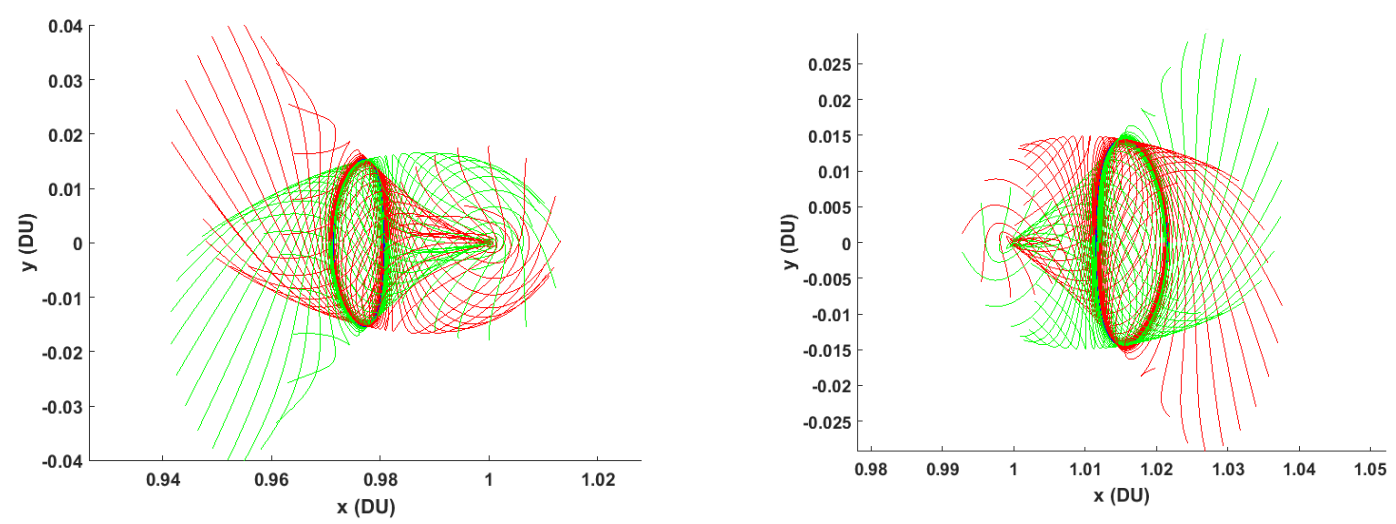

Figure 4.20: Manifolds propagated with 3 body dynamics from halos developed with 3 body dynamics

In all of the following figures (and the figures throughout the rest of this paper), the red lines represent the unstable manifolds, the green lines represent the stable manifolds, the solid blue line represents the predicted or desired halo orbit, and the dotted blue line represents what happens when the approximated halo is propagated forward in whatever dynamics are being used. For example, the halos in Fig. 4.20 were created and propagated with CR3BP dynamics, and those in the figures below were propagated with $\mathrm{N}$ body dynamics. As such, the propagation in the former shows no divergence from the predicted halo, but the latter cases diverge quickly and noticeably

Manifolds made from Richardson halo approximations behave erratically when propagated with CRNBP dynamics. The reasons for this should be mostly obvious. The perturbations from the other secondaries pulls the trajectile paths of a manifold in ways that are inconsistent with the properties of invariant manifolds in the three body 
system. Also, assuming the perturbation can be applied to the halo in the direction of the Jacobian at the associated Lagrange point, the technique for perturbing halos to create manifolds, is doubly false in the $\mathrm{N}$ body system. First, there is no well defined Lagrange point in the CRNBP, so even the halo is modeled to orbit around a unstable location, not just with different dynamics. Second, the Jacobian of the N body system is not the same as that of the 3 body system, meaning that the perturbations applied to the states are not applied in the correct direction, just an approximation of the correct direction. Take, for example, the Jupiter-Ganymede manifolds shown in Fig. 4.21. The halo used as the basis for these manifolds was developed in a 3 body system, placed into the $\mathrm{N}$ body system and the propagated The paths of the manifolds are wild and unpredictable, some paths lead into the planet, others diverge and head in the opposite direction as would be expect. Even the halo orbit itself diverges after a single orbit. The problems for manifolds produced around smaller secondaries are
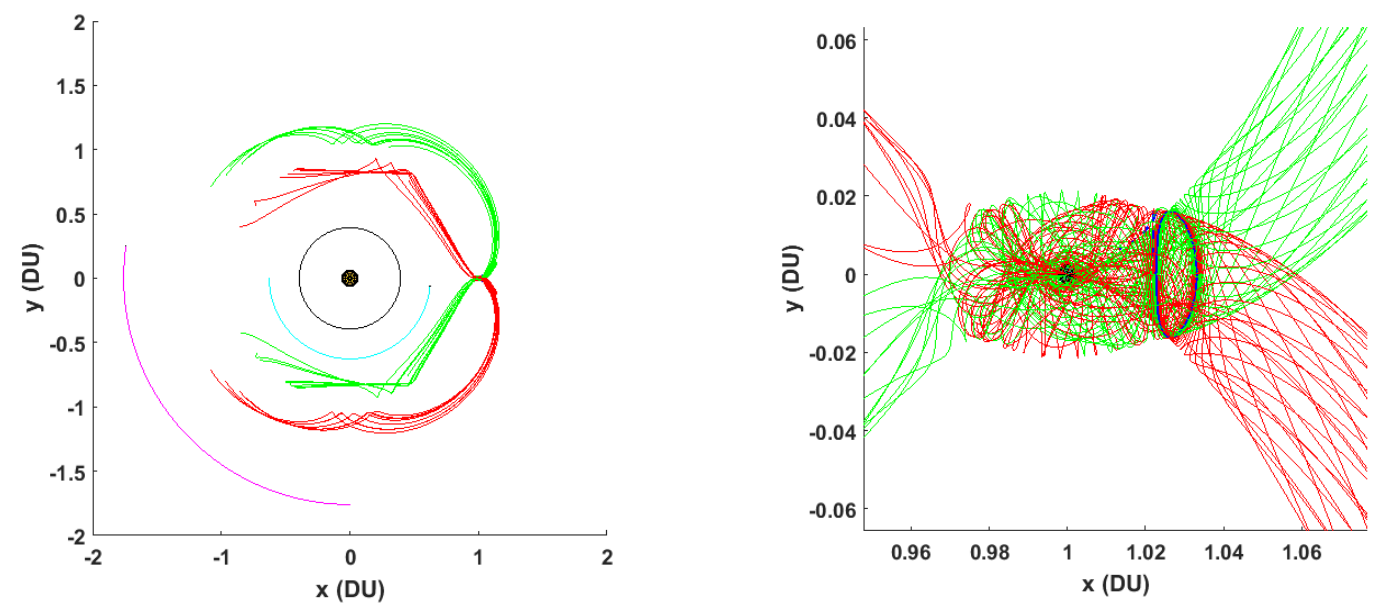

Figure 4.21: Manifolds propagated with $\mathrm{N}$ body dynamics from a halo developed with 3 body dynamics

even more disconcerting These manifolds immediately diverge from their expected trajectories and either fly away from the planet or careen into it. There are a whole host of possible reasons for this strange behavior - the most probable being that the halos shown and used to create the manifolds shown (Fig. 4.22 and Fig. 4.23) where 
not created in the same synodic frame as the one they were propagated forward in. Solving this would require translating the coordinates and equations of motion into a different rotational frame. The easiest way to do this would be to modify the N body system currently scaled to Ganymede and rescale it to the smaller secondaries. Once this was done, the results would have to be translated back into the Ganymede synodic frame. This is a non-trivial task and while it would be very interesting
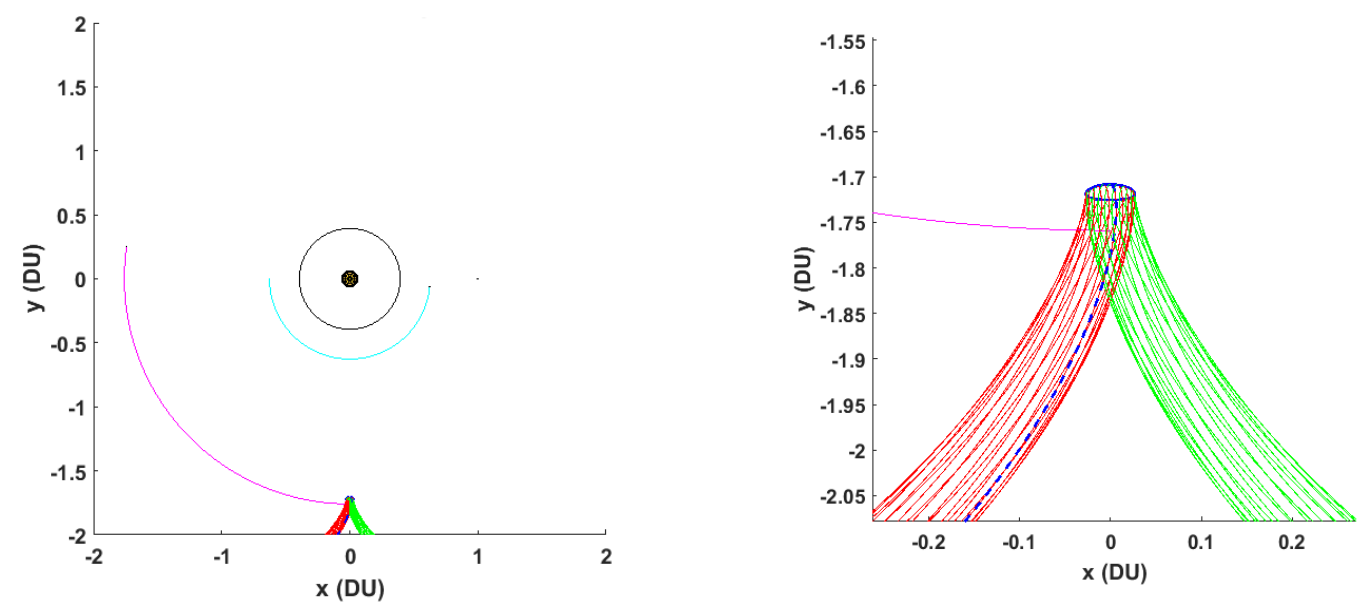

Figure 4.22: Manifolds propagated with $\mathrm{N}$ body dynamics from halos developed with 3 body dynamics near Callisto

and informative, it is not necessary for this thesis. It is likely that, since halos and manifolds can be produced around the smaller secondaries as shown above, that it would be possible to do this translation for the other moons as well. Instead, effort was focused on solving the issues that arose when 3 body manifolds around Ganymede were propagated with $\mathrm{N}$ body dynamics. If these problems could be solved, it is highly likely that the same process could then be applied to the other moons. To counteract these problems, halo orbits are instead approximated with $\mathrm{N}$ body dynamics. This not only makes the initial halo more stable in the system, it also means that the halo will be closer to orbiting its associated pseudo-Lagrange point. The problem of the Jacobian from the 3 body system applying perturbations in the wrong direction is avoided by simply decreasing the size of the initial perturbation. Since the Jacobian 

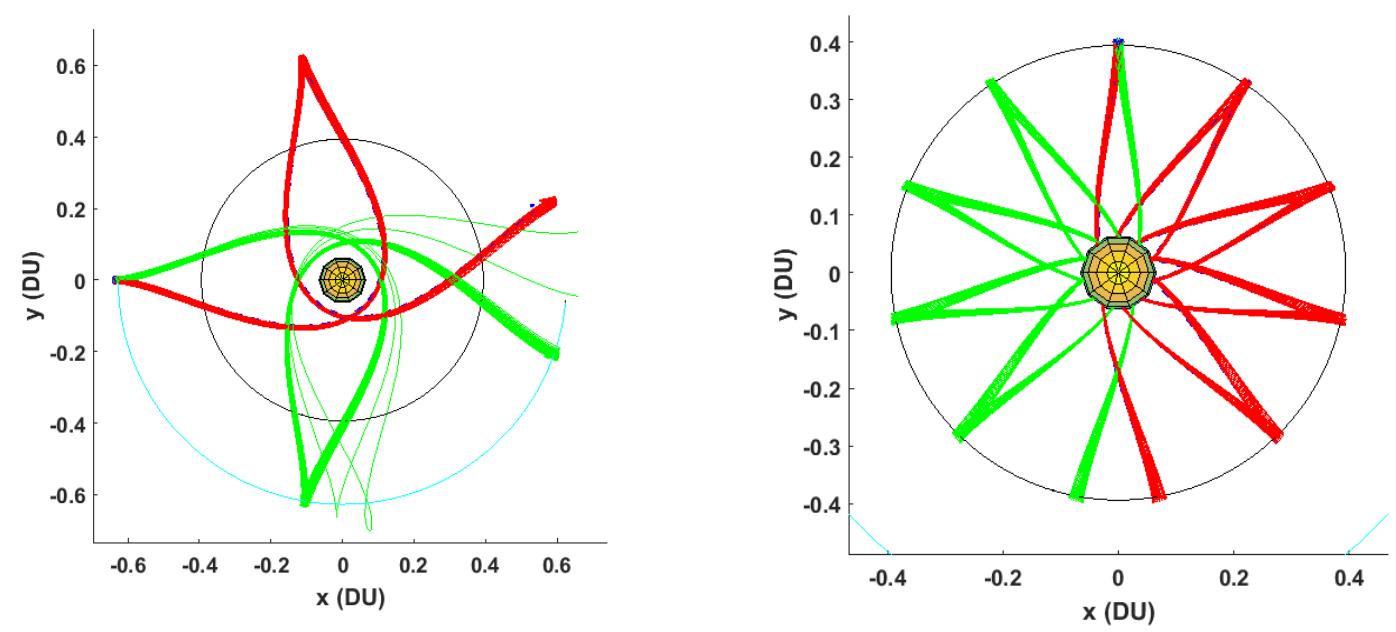

Figure 4.23: Manifolds propagated with $\mathrm{N}$ body dynamics from halos developed with 3 body dynamics near Europa and Io

of the $\mathrm{N}$ body system is the Jacobian of the 3 body system plus small perturbational terms from the extra secondaries, if the initial perturbation from the base halo is small enough, the pull from the indirect effect is reduced accordingly. Modifying the halos and manifolds in this manner proved successful, the results are shown in Fig. 4.24. The resulting manifolds are much cleaner and much more reminiscent of manifolds
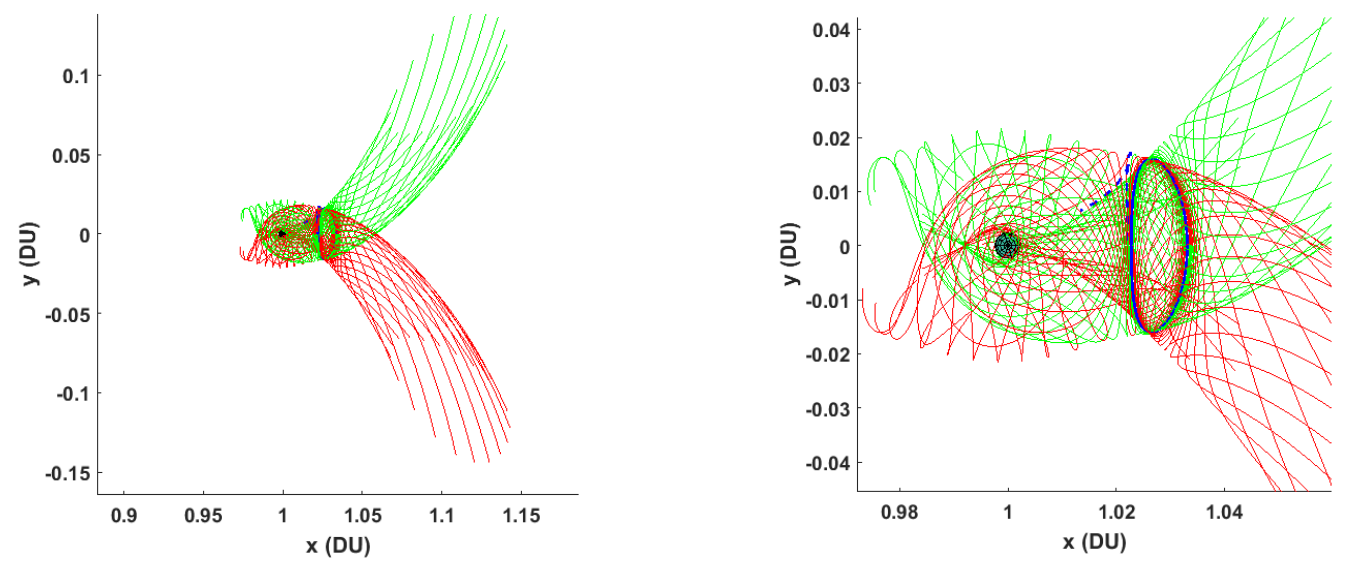

Figure 4.24: Manifolds propagated with $\mathrm{N}$ body dynamics from a halo developed with $\mathrm{N}$ body dynamics

from the CR3BP. There manifolds are not invariant, and it is likely that invariance is 
impossible with $\mathrm{N}$ bodies without correction or very unique conditions. Despite this, these manifolds are almost identical in appearance to invariant manifolds from the 3 body system and can be used in similar ways such as the construction of low energy trajectories.

\subsection{Low Energy Transfers and the Possibility of Highways}

This section deals with what was originally the inspiration for this thesis and what is perhaps the most interesting result of the analysis. Low energy trajectories exploit the unique dynamics of the CR3BP to make large orbital transfers with relatively little fuel. By utilizing the fact that manifolds will brisk the surface of the secondary body, manifolds from Sun-Planet interactions can be used to transfer to or from a planet's surface, e.g. to its moon(s). In the Jovian system, one could imagine how a Sun-Jupiter L1 manifold could be used to transfer to any of Jupiter's moons or, more precisely, any Halo orbit around any of Jupiter's moons. The idea behind "highways" is to find a series of these connections between manifolds so that a satellite could travel from halo to halo with almost no $\triangle v$. The properties of manifolds suggest that, if such manifold trajectories do exist, there must be similar trajectories in the opposite direction, meaning that there are paths on which continuous travel over huge distances and back is possible without much fuel. This section attempts to address the possibility of the existence of these paths.

The first step in solving for low energy trajectories is to set a up a nominal SunJupiter halo and it's associated manifolds. As shown in 4.25, both stable and unstable manifolds pass very close to the planet, and certainly pass within its direct sphere of influence. As is the case with figures in the previous section, the green manifolds represent stable manifolds, manifolds that converge onto a given halo, and red are unstable manifolds, manifolds that diverge from a given halo. The image on the left of 
the figure highlights relevant trajectories and the image on the right removes all trajectories that do not immediately pass close enough to Jupiter." Close enough" means closer than the average distance of Callisto from Jupiter, which is chosen simply due to that fact that the manifolds that come from Jupiter-Ganymede halos do not extend pass this orbit. Once this range of trajectories from the Sun-Jupiter (S-J) halo has
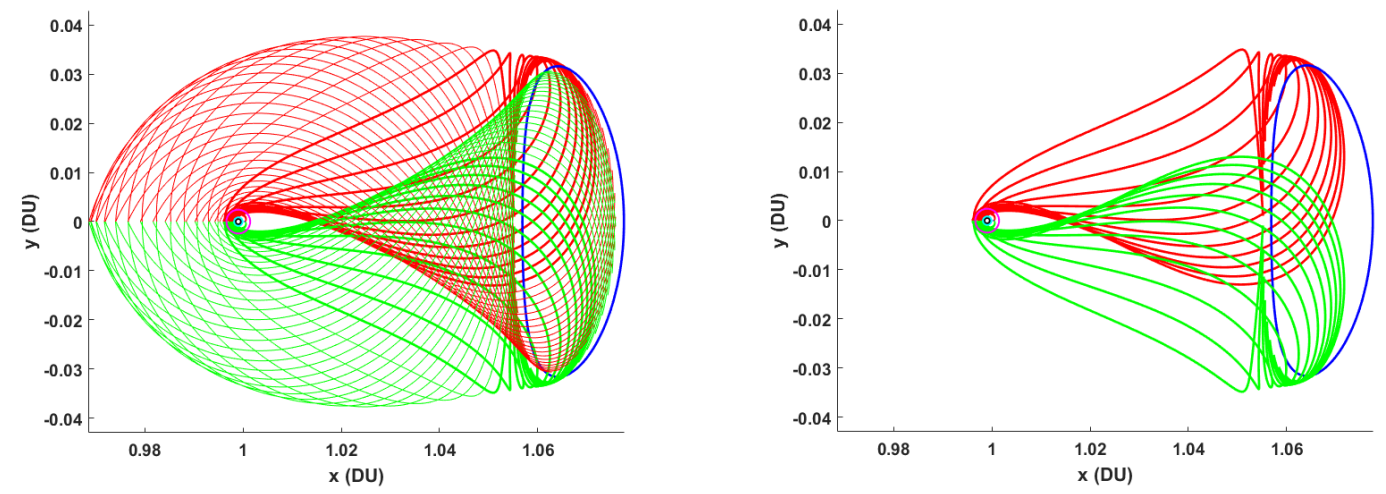

Figure 4.25: Low Energy Transfer into the Jovian System

been established, creating a set of manifolds for desired destination halo in the Jovian system can be constructed with similar size properties. Figure 4.26 zooms in on the first set and shows the nominal intersection of these manifold sets which then can be used to narrow down the possible trajectories even further. Mathematically speaking, there are an infinite number of possible solutions of trajectories that will travel along these manifolds from the S-J halo to the Jupiter-Ganymede (J-G) halo - irrespective of the initial geometric distribution of the Jovian moons due to the relatively small perturbations that the moons cause on the general shape of manifolds - but the number of functionally accessible trajectories is more limited. Because of this, the best looking trajectory is chosen as a starting point and then a simple optimization scheme can collapse on the appropriate trajectory that, literally, closes the loop. Instances where the initial and final halos have different z-excursions, have different phases, or belong to different classes were ignored for simplicity, making a solution for one particular instance relatively easy to find. The resulting trajectory is shown in Figures 
4.27 and 4.28. Assuming that the satellite starts on S-J halo, the satellite would first

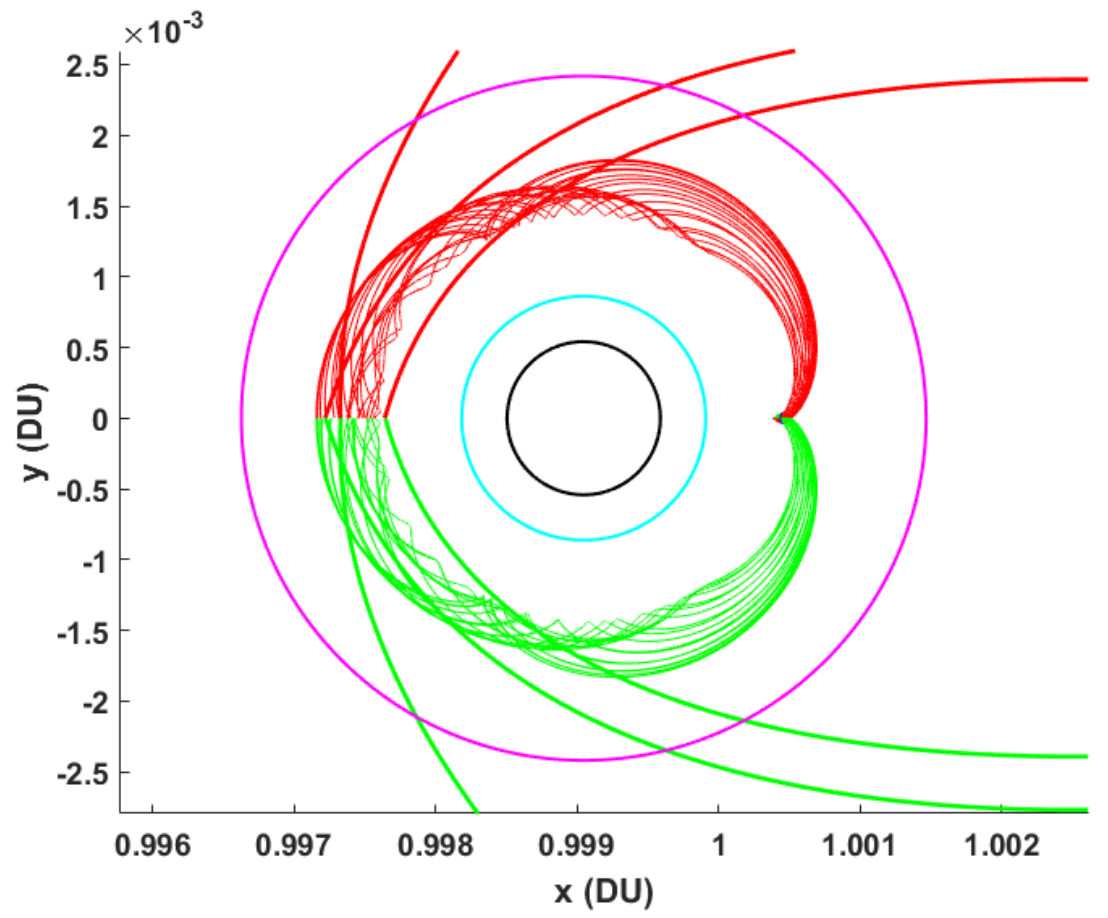

Figure 4.26: Low Energy Transfer into the Jovian System (zoom)

diverge along the unstable manifold, flying towards the planet. Once it reaches the intersection of the stable manifold from the J-G manifold, it would perform a burn to slow itself down to the proper speed, and then it would continue along the stable manifold until it reached the given J-G halo. This process is represented by the solid red and green lines in the figures below. If the fuel consumption of this burn is low enough, it would be feasible to make the same return trip along the corresponding mirrored manifold trajectories. This trajectory is given in the figures as a dotted line. It is important to note that this kind of trajectory will not work with systems that are rotating the same direction, e.g if the moons of Jupiter moved in a prograde fashion, this transfer would be much more difficult. This kind of transfer is also much more difficult when the two systems in question are not co-planar with one another, though this is less important due to the fact that the dynamics of these systems cause 


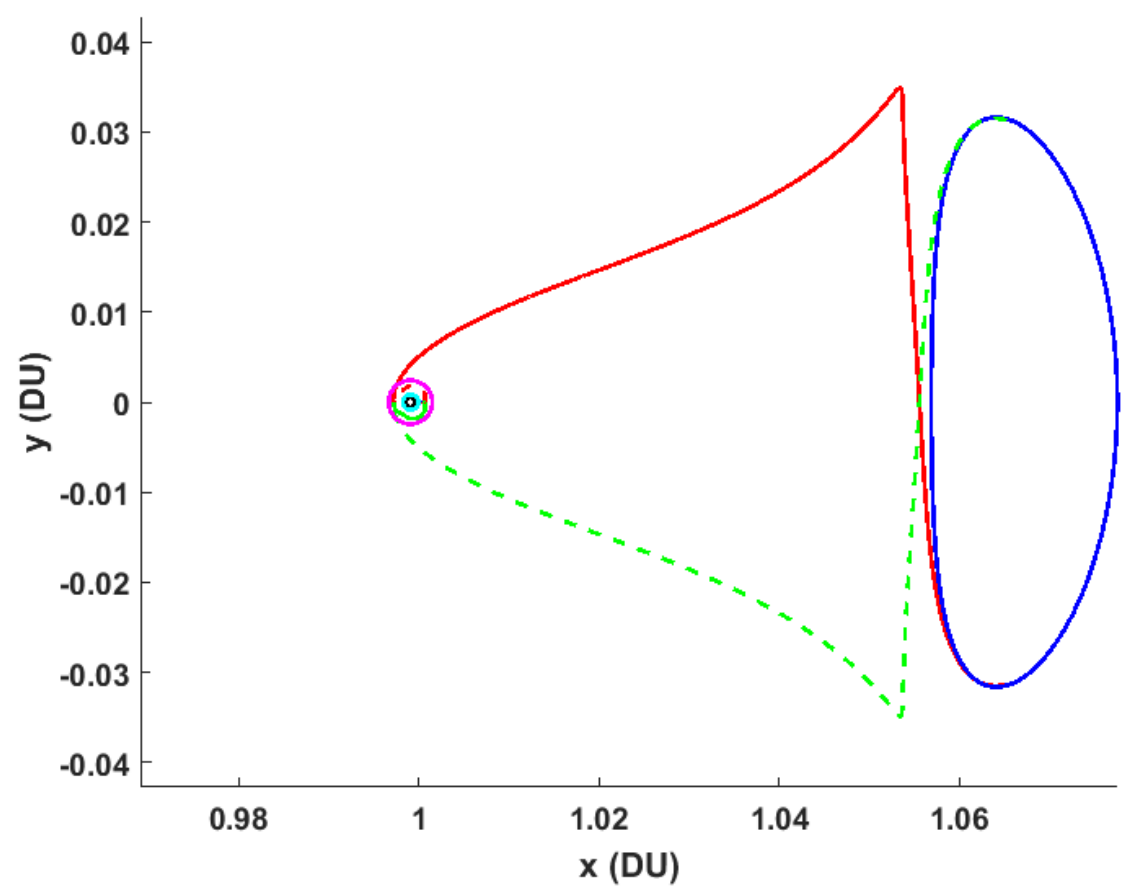

Figure 4.27: Transfer from Sun-Jupiter L1 Halo to Jupiter-Ganymede L2 Halo and back

regular sinusoidal oscillation through the x-y plane. It just so happens, however, that all of the Galilean moons are in retrograde, lowly inclined orbitransferred trajectories similar to the one shown potentially feasible for a mission. It is also important to note
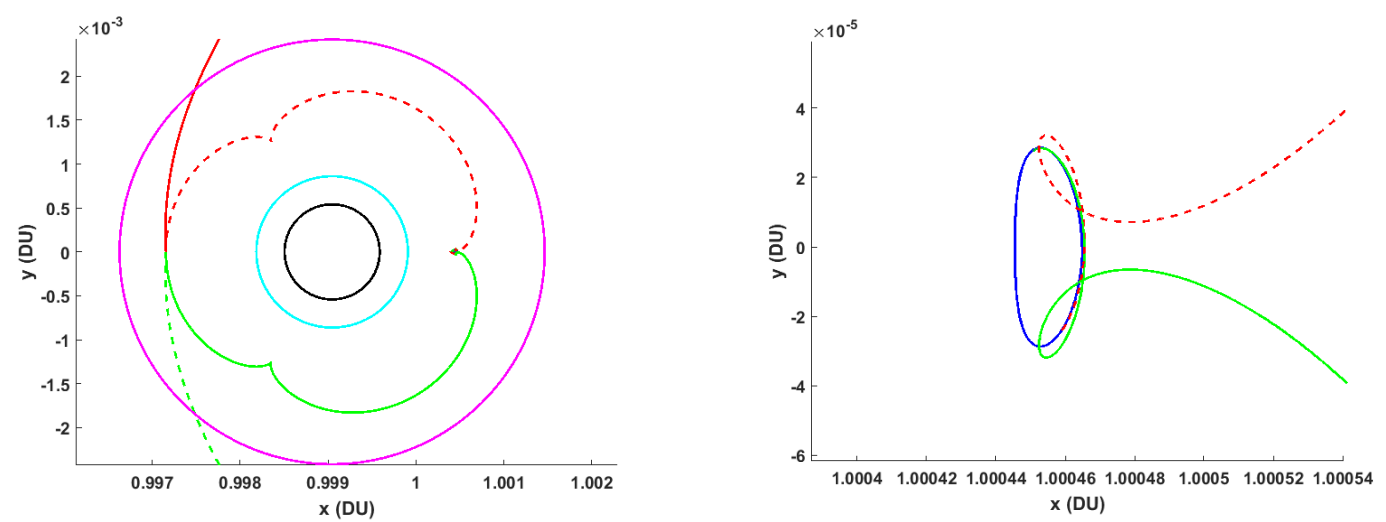

Figure 4.28: Transfer from Sun-Jupiter L1 Halo to Jupiter-Ganymede L2 Halo and back (zoom)

that the trajectory shown would not be mission feasible itself without a large amount 
of fuel (over $1 \frac{\mathrm{km}}{\mathrm{s}}$ ), it is simply a demonstration of the potential for these trajectories. There are many techniques for optimizing transfers and any number could be employed to try and make this trajectory more feasible. The possibility of this one orbit is relatively unimportant. It is important to recognize that these trajectories could be utilized in complex and interesting ways. Since it is only a question of which of the $\mathrm{N}$ bodies should be transfered to, and since it has been shown that manifolds can be used to transfer between L1 and L2 points around a body, one can easily imagine a string of different manifold connections going from the Sun-Jupiter trajectories to Jupiter-Callisto trajectories to Jupiter-Ganymede trajectories and so on. Perhaps if combined with aerocapture maneuvers or other similar flyby techniques, the ideas could eventually be pushed to a realistic framework. 


\section{Chapter 5}

\section{FUTURE WORK}

The analysis done here opens up the possibility of a plethora of future projects. The first task that needs to be approached deals with the numerical accuracy of this method, the ability of this method to accurately propagate states in applicable systems, not just its ability to predict trends, and the sensitivity of this system to different geometries, mass ratios, and perturbations, such as that caused by the pull of the sun. Analyzing these problems would push this system towards (or away from) more practical applications. The effects of the mass ratio on the validity of the assumptions of the CRNBP is only discussed enough to prove the assumptions for the Jovian system, but it is also important to understand more generally when these assumptions break down. There is also a possibility that unique geometric configurations could lead to unique energy contours, trajectories, and transfers. It

will also be important to examine the perturbations caused by the eccentricity of orbits. A lot of work has been done on this problem for the CR3BP and it seems that this perturbation is one of the largest sources of error. It is probable that this is also the case for the CRNBP and needs to be examined thoroughly.

Other projects suggested by this paper include modeling other planets. The results of this paper are limited to the Jovian system exclusively. Both the solar system and the Saturnian system have similar mass properties as the Jovian system, making them viable candidates for this kind of analysis. Saturn would be difficult due to the fact that it would be necessary to include the perturbations from the planet's rings. The solar system could be analyzed to look at the possibility of using these models for interplanetary transfers, with low energy trajectories as their basis. Surprisingly, Uranus also fits nicely under the assumptions required for the CRNBP equations. 
With the interest in creating orbiters around the system rising in recent years, these equations could be the first step in higher level mission analysis.

The results from the derivation of the Jacobi constant are consistent with the 3 body system, but they are not examine at length in this paper. It would be worthwhile to examine the Jacobi energy of different systems as well as looking the motion and trends of the energy contours as the different moons in the system process. Another potential study could look at the variance and stability of the points of minimum acceleration in the system for different planetary geometries and under the effect of perturbations.

This thesis also opened up the possibility of a new examination of halo orbits, manifolds, and low energy trajectories that was only brisked. It might be possible to find a lower order solution to periodic orbits in the CRNBP, similar to the solution found by Richardson for halo orbits in the three body problem, and it might be possible to find a way of converging on more accurate solutions using the state transition matrix, similar to the solution developed by Howell. The solution to correct halo orbits in the CRNBP was a simplistic solution that did not account for a number of different and applicable trajectory correction methods. Higher fidelity correction optimization, and integrated thruster capabilities and control would both be interesting studies with practical results. Similarly, the low energy transfer demonstrated was simple and did not have any kind of optimization. To make more concrete conclusions about the possibility and mission feasibility of these trajectories, there needs to be a more inclusive look at how these trajectories could be accomplished. 


\section{Chapter 6}

\section{CONCLUSION}

This thesis is a brief look at a new solution to a problem that has been approached in many different ways in the past - the $\mathrm{N}$ body problem. By focusing on planetary systems, satellite dynamics can be modeled in a fashion similar to the Circular Restricted Three Body Problem with the Circular Restricted N Body Problem. It was found that this new formulation of the dynamics can then utilize the tools created from all the research into the CR3BP to reassess the possibility of different complex trajectories in systems where there are more than just two large gravitational bodies affecting the dynamics, namely periodic and semi-periodic orbits, halo orbits, and low energy transfers It was also found that not only system dynamics, but models of the Jacobi constant could also be formulated similarly to the CR3BP.

Validating the authenticity of these new sets of equations, the CRNBP dynamics are applied to a satellite in the Earth-Moon system and compared to a simulation of the CR3BP under identical circumstances. This test verified the dynamics of the CRNBP, showing that the two systems created almost identical results with relatively small deviations over time and with essentially identical path trends. In the Jovian system, it was found the mass ratio required to validated the assumptions required to integrate the equations of motion was around .1

This demonstration is simplistic and, in the end, merely academic in its current state, but it opens up the possibility of considering the trajectories of satellites in systems with N major gravitational bodies without forcing highly idealized dynamics. Even with the constant influence of multiple bodies, it seems that a body's dynamics

are relatively unchanged and trend in very similar ways to more idealized cases like the CR3BP. The equations and methods here are another step towards a better 
understanding of $\mathrm{N}$ body dynamics and give a cursory glance at the possibility of results of applying them to a real world system. This is just a start, but a door has been opened to a new way of looking at an age old problem. 


\section{BIBLIOGRAPHY}

[1] G. Archambeau, P. Augros, and E. Trelat. Eight-shaped lissajous orbits in the earth-moon system. MathematicS In Action, Vol. 4:1-23, 2011.

[2] R. Bate, D. Mueller, and J. White. Fundamentals of astrodynamics. Dover Publications, Inc., 1971.

[3] N. Bosanac, J. E. Marsden, A. Moore, and S. Campagnola. Titan trajectory design using invariant manifolds and resonant gravity assists. Technical Report No. 136, American Astonomical Society, November 2010.

[4] W. Dehnen and J. Read. N-body simulations of gravitational dynamics. Technical report, Department of Physics and Astronomy, University of Leicester, United Kingdom, and Department of Physics, Institute for Astronomy, Zurich, May 2011.

[5] K. Howel and H. J. Pernicka. Numerical determination of lissajous trajectories in the restricted three-body problem. Technical report, School of Aeronautics and Astronautics, Purdue University, July 1987.

[6] J. S. Parker. Low-energy ballistic lunar transfers. Technical report, Aerospace Engineering Sciences, University of Colorado, October 2003.

[7] J. S. Parker and R. L. Anderson. Low-energy lunar trajectory design. Technical report, Jet Propulsion Labratory, October 1996.

[8] D. L. Richardson. Analytic construction of periodic orbits about the collinear points. Technical report, Department of Aerospace Engineering, University of Cincinnati, August 1979. 
[9] C. E. Roberts. The soho mission 11 halo orbit recovery from the attitude control anomalies of 1998. Technical report, Libration Point Orbits and Applications Conference Parador dAiguablava, Girona, Spain, June 2002.

[10] R. Thurman and P. A. Worfolk. The geometry of halo orbits in the circular restricted three-body problem. Technical report, The Geometry Center, University of Minnesota, October 1996.

[11] D. A. Vallado and W. D. McClain. Fundamentals of astrodynamics and applications. Kluwer Academic Publishers, 2001. 